\title{
CLUSTER-TILTED AND QUASI-TILTED ALGEBRAS
}

\author{
IBRAHIM ASSEM, RALF SCHIFFLER, AND KHRYSTYNA SERHIYENKO
}

\begin{abstract}
In this paper, we prove that relation-extensions of quasitilted algebras are 2-Calabi-Yau tilted. With the objective of describing the module category of a cluster-tilted algebra of euclidean type, we define the notion of reflection so that any two local slices can be reached one from the other by a sequence of reflections and coreflections. We then give an algorithmic procedure for constructing the tubes of a cluster-tilted algebra of euclidean type. Our main result characterizes quasi-tilted algebras whose relation-extensions are cluster-tilted of euclidean type.
\end{abstract}

\section{INTRODUCTION}

Cluster-tilted algebras were introduced by Buan, Marsh and Reiten [BMR] and, independently in [CCS] for type $\mathbb{A}$ as a byproduct of the now extensive theory of cluster algebras of Fomin and Zelevinsky [FZ]. Since then, cluster-tilted algebras have been the subject of several investigations, see, for instance, [ABCP, ABS, BFPPT, BT, BOW, BMR2, KR, OS, ScSe, ScSe2].

In particular, in $[\mathrm{ABS}]$ is given a construction procedure for cluster-tilted algebras: let $C$ be a triangular algebra of global dimension two over an algebraically closed field $k$, and consider the $C$-C -bimodule $\operatorname{Ext}_{C}^{2}(D C, C)$, where $D=\operatorname{Hom}_{k}(-, k)$ is the standard duality, with its natural left and right $C$-actions. The trivial extension of $C$ by this bimodule is called the relation-extension $\widetilde{C}$ of $C$. It is shown there that, if $C$ is tilted, then its relation-extension is cluster-tilted, and every cluster-tilted algebra occurs in this way.

Our purpose in this paper is to study the relation-extensions of a wider class of triangular algebras of global dimension two, namely the class of quasi-tilted algebras, introduced by Happel, Reiten and Smalø in [HRS]. In general, the relation-extension of a quasi-tilted algebra is not cluster-tilted, however it is 2-Calabi-Yau tilted, see Theorem 3.1 below. We then look more closely at those cluster-tilted algebras which are tame and representationinfinite. According to [BMR], these coincide exactly with the cluster-tilted algebras of euclidean type. We ask then the following question: Given a cluster-tilted algebra $B$ of euclidean type, find all quasi-tilted algebras $C$

The first author gratefully acknowledges partial support from the NSERC of Canada. The second author was supported by the NSF CAREER grant DMS-1254567 and by the University of Connecticut. The third author was supported by the NSF Postdoctoral fellowship MSPRF-1502881. 
such that $B=\widetilde{C}$. A similar question has been asked (and answered) in [ABS2], where, however, $C$ was assumed to be tilted.

For this purpose, we generalize the notion of reflections of [ABS4]. We prove that this operation allows to produce all tilted algebras $C$ such that

$B=\widetilde{C}$, see Theorem 4.11. In [ABS4] this result was shown only for clustertilted algebras of tree type. We also prove that, unlike those of [ABS4], reflections in the sense of the present paper are always defined, that the reflection of a tilted algebra is also tilted of the same type, and that they have the same relation-extension, see Theorem 4.4 and Proposition 4.8 below. Because all tilted algebras having a given cluster-tilted algebra as relationextension are given by iterated reflections, this gives an algorithmic answer to our question above.

After that, we look at the tubes of a cluster-tilted algebra of euclidean type and give a procedure for constructing those tubes which contain a projective, see Proposition 5.6.

We then return to quasi-tilted algebras in our last section, namely we define a particular two-sided ideal of a cluster-tilted algebra, which we call the partition ideal. Our first result (Theorem 6.1) shows that the quasitilted algebras which are not tilted but have a given cluster-tilted algebra $B$ of euclidean type as relation-extension are the quotients of $B$ by a partition ideal. We end the paper with the proof of our main result (Theorem 6.3) which says that if $C$ is quasi-tilted and such that $B=\widetilde{C}$, then either $C$ is the quotient of $B$ by the annihilator of a local slice (and then $C$ is tilted) or it is the quotient of $B$ by a partition ideal (and then $C$ is not tilted except in two cases easy to characterize).

\section{Preliminaries}

2.1. Notation. Throughout this paper, algebras are basic and connected finite dimensional algebras over a fixed algebraically closed field $k$. For an algebra $C$, we denote by $\bmod C$ the category of finitely generated right $C$ modules. All subcategories are full, and identified with their object classes. Given a category $\mathcal{C}$, we sometimes write $M \in \mathcal{C}$ to express that $M$ is an object in $\mathcal{C}$. If $\mathcal{C}$ is a full subcategory of $\bmod C$, we denote by add $\mathcal{C}$ the full subcategory of $\bmod C$ having as objects the finite direct sums of summands of modules in $\mathcal{C}$.

For a point $x$ in the ordinary quiver of a given algebra $C$, we denote by $P(x), I(x), S(x)$ respectively, the indecomposable projective, injective and simple $C$-modules corresponding to $x$. We denote by $\Gamma(\bmod C)$ the Auslander-Reiten quiver of $C$ and by $\tau=D \operatorname{Tr}, \tau^{-1}=\operatorname{Tr} D$ the AuslanderReiten translations. For further definitions and facts, we refer the reader to [ARS, ASS, S].

2.2. Tilting. Let $Q$ be a finite connected and acyclic quiver. A module $T$ over the path algebra $k Q$ of $Q$ is called tilting if $\operatorname{Ext}_{k Q}^{1}(T, T)=0$ and the number of isoclasses (isomorphism classes) of indecomposable summands of 
$T$ equals $\left|Q_{0}\right|$, see [ASS]. An algebra $C$ is called tilted of type $Q$ if there exists a tilting $k Q$-module $T$ such that $C=\operatorname{End}_{k Q} T$. It is shown in [Ri] that an algebra $C$ is tilted if and only if it contains a complete slice $\Sigma$, that is, a finite set of indecomposable modules such that

1) $\bigoplus_{U \in \Sigma} U$ is a sincere $C$-module.

2) If $U_{0} \rightarrow U_{1} \rightarrow \cdots \rightarrow U_{t}$ is a sequence of nonzero morphisms between indecomposable modules with $U_{0}, U_{t} \in \Sigma$ then $U_{i} \in \Sigma$ for all $i$ (convexity).

3) If $M$ is an indecomposable non-projective $C$-module then at most one of $M, \tau M$ belongs to $\Sigma$.

4) If $M, S$ are indecomposable $C$-modules, $f: M \rightarrow S$ an irreducible morphism and $S \in \Sigma$, then either $M \in \Sigma$ or $M$ is non-injective and $\tau^{-1} M \in \Sigma$.

For more on tilting and tilted algebras, we refer the reader to [ASS].

Tilting can also be done within the framework of a hereditary category. Let $\mathcal{H}$ be an abelian $k$-category which is Hom-finite, that is, such that, for all $X, Y \in \mathcal{H}$, the vector space $\operatorname{Hom}_{\mathcal{H}}(X, Y)$ is finite dimensional. We say that $\mathcal{H}$ is hereditary if $\operatorname{Ext}_{\mathcal{H}}^{2}(-, ?)=0$. An object $T \in \mathcal{H}$ is called a tilting object if $\operatorname{Ext}_{\mathcal{H}}^{1}(T, T)=0$ and the number of isoclasses of indecomposable objects of $T$ is the rank of the Grothendieck group $K_{0}(\mathcal{H})$.

The endomorphism algebras of tilting objects in hereditary categories are called quasi-tilted algebras. For instance, tilted algebras but also canonical algebras (see $[\mathrm{Ri}]$ ) are quasi-tilted. Quasi-tilted algebras have attracted a lot of attention and played an important role in representation theory, see for instance [HRS, Sk].

2.3. Cluster-tilted algebras. Let $Q$ be a finite, connected and acyclic quiver. The cluster category $\mathcal{C}_{Q}$ of $Q$ is defined as follows, see [BMRRT]. Let $F$ denote the composition $\tau_{\mathcal{D}}^{-1}[1]$, where $\tau_{\mathcal{D}}^{-1}$ denotes the inverse AuslanderReiten translation in the bounded derived category $\mathcal{D}=\mathcal{D}^{b}(\bmod k Q)$, and [1] denotes the shift of $\mathcal{D}$. Then $\mathcal{C}_{Q}$ is the orbit category $\mathcal{D} / F$ : its objects are the $F$-orbits $\widetilde{X}=\left(F^{i} X\right)_{i \in \mathbb{Z}}$ of the objects $X \in \mathcal{D}$, and the space of morphisms from $\widetilde{X}=\left(F^{i} X\right)_{i \in \mathbb{Z}}$ to $\tilde{Y}=\left(F^{i} Y\right)_{i \in \mathbb{Z}}$ is

$$
\operatorname{Hom}_{\mathcal{C}_{Q}}(\tilde{X}, \tilde{Y})=\bigoplus_{i \in \mathbb{Z}} \operatorname{Hom}_{\mathcal{D}}\left(X, F^{i} Y\right)
$$

Then $\mathcal{C}_{Q}$ is a triangulated category with almost split triangles and, moreover, for $\widetilde{X}, \widetilde{Y} \in \mathcal{C}_{Q}$ we have a bifunctorial isomorphism $\operatorname{Ext}_{\mathcal{C}_{Q}}^{1}(\widetilde{X}, \widetilde{Y}) \cong$ $\operatorname{DExt}_{\mathcal{C}_{Q}}^{1}(\widetilde{Y}, \widetilde{X})$. This is expressed by saying that the category $\mathcal{C}_{Q}$ is 2 -CalabiYau. 
An object $\widetilde{T} \in \mathcal{C}_{Q}$ is called tilting if $\operatorname{Ext}_{\mathcal{C}_{Q}}^{1}(\widetilde{T}, \widetilde{T})=0$ and the number of isoclasses of indecomposable summands of $\widetilde{T}$ equals $\left|Q_{0}\right|$. The endomorphism algebra $B=\operatorname{End}_{\mathcal{C}_{Q}} \widetilde{T}$ is then called cluster-tilted of type $Q$. More generally, the endomorphism algebra $\operatorname{End}_{\mathcal{C}} \widetilde{T}$ of a tilting object $\widetilde{T}$ in a 2-CalabiYau category with finite dimensional Hom-spaces is called a 2-Calabi-Yau tilted algebra, see [Re].

Let now $T$ be a tilting $k Q$-module, and $C=\operatorname{End}_{k Q} T$ the corresponding tilted algebra. Then it is shown in $[\mathrm{ABS}]$ that the trivial extension $\widetilde{C}$ of $C$ by the $C$ - $C$-bimodule $\operatorname{Ext}_{C}^{2}(D C, C)$ with the two natural actions of $C$, the so-called relation-extension of $C$, is cluster-tilted. Conversely, if $B$ is cluster-tilted, then there exists a tilted algebra $C$ such that $B=\widetilde{C}$.

Recall that a path $X_{0} \rightarrow X_{1} \rightarrow \cdots \rightarrow X_{t}$ in the Auslander-Reiten quiver is called sectional if $\tau X_{i+1} \neq X_{i-1}$ for all $i=1,2, \ldots, n-1$, see for example [ASS, page 361].

Let now $B$ be a cluster-tilted algebra, then a full subquiver $\Sigma$ of $\Gamma(\bmod B)$ is a local slice, see [ABS2], if:

1) $\Sigma$ is a presection, that is, if $X \rightarrow Y$ is an arrow then:

(a) $X \in \Sigma$ implies that either $Y \in \Sigma$ or $\tau Y \in \Sigma$

(b) $Y \in \Sigma$ implies that either $X \in \Sigma$ or $\tau^{-1} X \in \Sigma$.

2) $\Sigma$ is sectionally convex, that is, if $X=X_{0} \rightarrow X \rightarrow \cdots \rightarrow X_{t}=Y$ is a sectional path in $\Gamma(\bmod B)$ then $X, Y \in \Sigma$ implies that $X_{i} \in \Sigma$ for all $i$.

3) $\left|\Sigma_{0}\right|=\operatorname{rk} K_{0}(B)$.

Let $C$ be tilted, then, under the standard embedding $\bmod C \rightarrow \bmod \widetilde{C}$, any complete slice in the tilted algebra $C$ embeds as a local slice in $\bmod \widetilde{C}$, and any local slice in $\bmod \widetilde{C}$ occurs in this way. If $B$ is a cluster-tilted algebra, then a tilted algebra $C$ is such that $B=\widetilde{C}$ if and only if there exists a local slice $\Sigma$ in $\Gamma(\bmod B)$ such that $C=B / \operatorname{Ann}_{B} \Sigma$, where $\operatorname{Ann}_{B} \Sigma=$ $\bigcap_{X \in \Sigma} \operatorname{Ann}_{B} X$, see $[\mathrm{ABS} 2]$.

Let $\Sigma$ be a local slice in the transjective component of $\Gamma(\bmod B)$ having the property that all the sources in $\Sigma$ are injective $B$-modules. Then $\Sigma$ is called a rightmost slice of $B$. Let $x$ be a point in the quiver of $B$ such that $I(x)$ is an injective source of the rightmost slice $\Sigma$. Then $x$ is called a strong sink. Leftmost slices and strong sources are defined dually.

\section{From quasi-Tilted to Cluster-Tilted algebras}

We start with a motivating example. Let $C$ be the tilted algebra of type $\widetilde{\mathbb{A}}$ given by the quiver

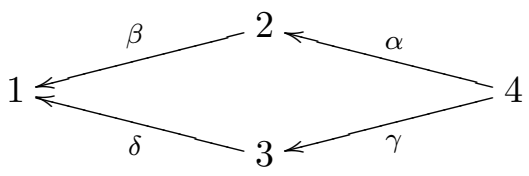


bound by $\alpha \beta=0, \gamma \delta=0$. Its relation-extension is the cluster-tilted algebra $B$ given by the quiver

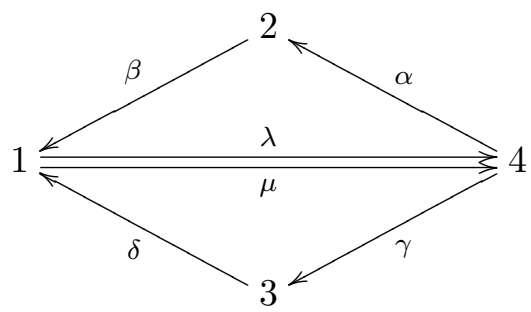

bound by $\alpha \beta=0, \beta \lambda=0, \lambda \alpha=0, \gamma \delta=0, \delta \mu=0, \mu \gamma=0$. However, $B$ is also the relation-extension of the algebra $C^{\prime}$ given by the quiver

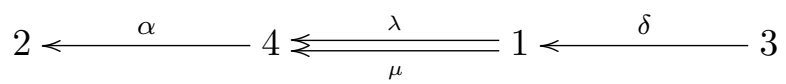

bound by $\lambda \alpha=0, \delta \mu=0$. This latter algebra $C^{\prime}$ is not tilted, but it is quasitilted. In particular, it is triangular of global dimension two. Therefore, the question arises naturally whether the relation-extension of a quasi-tilted algebra is always cluster-tilted. This is certainly not true in general, for the relation-extension of a tubular algebra is not cluster-tilted see Remark 3.2 below. However, it is 2-Calabi-Yau tilted. In this section, we prove that the relation-extension of a quasi-tilted algebra is always 2-Calabi-Yau tilted.

Let $\mathcal{H}$ be a hereditary category with tilting object $T$. Due to $[\mathrm{H}$, Theorem 3.1], there exist an algebra $A$, which is hereditary or canonical, and a triangle equivalence $\Phi: \mathcal{D}^{b}(\mathcal{H}) \rightarrow \mathcal{D}^{b}(\bmod A)$. Let $T^{\prime}$ denote the image of $T$ under this equivalence. Because $\Phi$ preserves the shift and the AuslanderReiten translation, it induces an equivalence between the cluster categories $\mathcal{C}_{\mathcal{H}}$ and $\mathcal{C}_{A}$, see [Am, Section 4.1]. Indeed, because $A$ is canonical or hereditary, it follows that $\mathcal{C}_{A} \cong \mathcal{D}^{b}(\bmod A) / F$, where $F=\tau^{-1}[1]$. Therefore, we have $\operatorname{End}_{\mathcal{C}_{\mathcal{H}}} T \cong \operatorname{End}_{\mathcal{C}_{A}} T^{\prime}$.

We say that a 2-Calabi-Yau tilted algebra $\operatorname{End}_{\mathcal{C}} T$ is of canonical type if the 2-Calabi-Yau category $\mathcal{C}$ is the cluster category of a canonical algebra. The proof of the next theorem follows closely the proof of Theorem 3.4 in [ABS].

Theorem 3.1. Let $C$ be a quasi-tilted algebra. Then its relation-extension $\widetilde{C}$ is cluster-tilted or it is 2-Calabi-Yau tilted of canonical type.

Proof. Because $C$ is quasi-tilted, there exist a hereditary category $\mathcal{H}$ and a tilting object $T$ in $\mathcal{H}$ such that $C=\operatorname{End}_{\mathcal{H}} T$. As observed above, there exist an algebra $A$, which is hereditary or canonical, and a triangle equivalence $\Phi$ : $\mathcal{D}^{b}(\mathcal{H}) \rightarrow \mathcal{D}^{b}(\bmod A)$. Let $T^{\prime}=\Phi(T)$. We have $\mathcal{D}^{b}(\bmod C) \cong \mathcal{D}^{b}(\bmod A) \cong$ 
$\mathcal{D}^{b}(\mathcal{H})$, and therefore

$$
\begin{aligned}
\operatorname{Ext}_{C}^{2}(D C, C) & \cong \operatorname{Hom}_{\mathcal{D}^{b}(\bmod C)}(\tau C[1], C[2]) \\
& \cong \operatorname{Hom}_{\mathcal{D}^{b}(\mathcal{H})}(\tau T[1], T[2]) \\
& \cong \operatorname{Hom}_{\mathcal{D}^{b}(\mathcal{H})}\left(T, \tau^{-1} T[1]\right) \\
& \cong \operatorname{Hom}_{\mathcal{D}^{b}(\mathcal{H})}(T, F T)
\end{aligned}
$$

Thus the additive structure of $C \ltimes \operatorname{Ext}_{C}^{2}(D C, C)$ is that of

$$
\begin{aligned}
C \oplus \operatorname{Ext}_{C}^{2}(D C, C) & \cong \operatorname{End}_{\mathcal{H}}(T) \oplus \operatorname{Hom}_{\mathcal{D}^{b}(\mathcal{H})}(T, F T) \\
& \cong \oplus_{i \in \mathbb{Z}} \operatorname{Hom}_{\mathcal{D}^{b}(\mathcal{H})}(T, F T) \\
& \cong \operatorname{Hom}_{\mathcal{C}_{\mathcal{H}}}(T, T) \\
& \cong \operatorname{End}_{\mathcal{C}_{\mathcal{H}}} T .
\end{aligned}
$$

Then, we check exactly as in [ABS, Section 3.3] that the multiplicative structure is preserved. This completes the proof.

Remark 3.2. The theorem implies that the relation-extension of a tubular algebra is not cluster-tilted. Indeed, the derived category of a tubular algebra, and hence its cluster category, does not have a transjective component $[\mathrm{HR}]$, and therefore the 2-Calabi-Yau tilted algebras that arise from this cluster category, so in particular the relation extensions of tubular algebras, also do not have a transjective component. On the other hand, cluster-tilted algebras always have a transjective component.

Let $C$ be a representation-infinite quasi-tilted algebra. Then $C$ is derived equivalent to a hereditary or a canonical algebra $A$. Let $n_{A}$ denote the tubular type of $A$. We then say that $C$ has canonical type $n_{C}=n_{A}$.

Lemma 3.3. Let $C$ be a representation-infinite quasi-tilted. Then its relationextension $\widetilde{C}$ is cluster-tilted of euclidean type if and only if $n_{C}$ is one of

$$
(p, q),(2,2, r),(2,3,3),(2,3,4),(2,3,5), \text { with } p \leq q, 2 \leq r .
$$

Proof. Indeed, $\widetilde{C}$ is cluster-tilted of euclidean type if and only if $C$ is derived equivalent to a tilted algebra of euclidean type, and this is the case if and only if $n_{C}$ belongs to the above list.

Remark 3.4. It is possible that $C$ is domestic, [Sk, Section 1], but yet $\widetilde{C}$ is wild. Indeed, we modify the example after Corollary D in [Sk]. Recall from [Sk] that there exists a tame concealed full convex subcategory $K$ such that $C$ is a semiregular branch enlargement of $K$

$$
C=\left[E_{i}\right] K\left[F_{j}\right]
$$

where $E_{i}, F_{j}$ are (truncated) branches. Then the representation theory of $C$ is determined by those of $C^{-}=\left[E_{i}\right] K$ and $C^{+}=K\left[F_{j}\right]$. Let $C$ be given by the quiver 


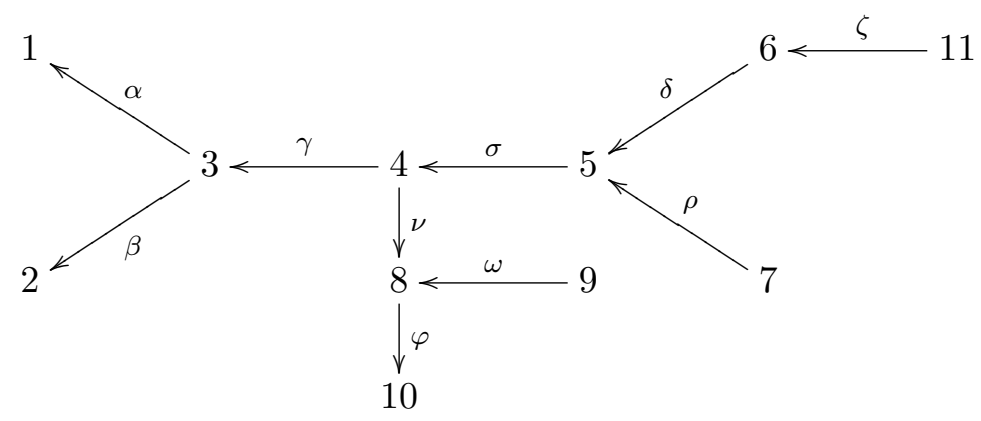

bound by the relations $\sigma \nu=0, \omega \varphi=0, \zeta \delta \sigma \gamma \beta=0$. Here $C^{-}$is the full subcategory generated by $C_{0} \backslash\{11\}$ and $C^{+}$the one generated by $C_{0} \backslash$ $\{8,9,10\}$. Then $C^{-}$has domestic tubular type $(2,2,7)$ and $C^{+}$has domestic tubular type $(2,3,4)$. Therefore $C$ is domestic. On the other hand, the canonical type of $C$ is $(2,3,7)$, which is wild. In this example, the 2-CalabiYau tilted algebra $\widetilde{C}$ is not cluster-tilted, because it is not of euclidean type, but the derived category of $\bmod C$ contains tubes, see $[\mathrm{R}]$.

Remark 3.5. There clearly exist algebras which are not quasi-tilted but whose relation-extension is cluster-tilted of euclidean type. Indeed, let $C$ be given by the quiver

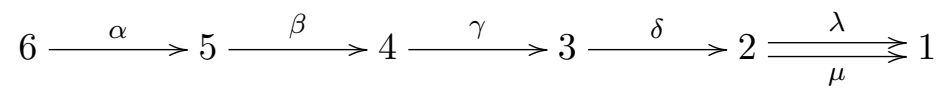

bound by $\alpha \beta=0, \delta \lambda=0$. Then $C$ is iterated tilted of type $\widetilde{\mathbb{A}}$ of global dimension 2, see $[\mathrm{FPT}]$. Its relation-extension is given by

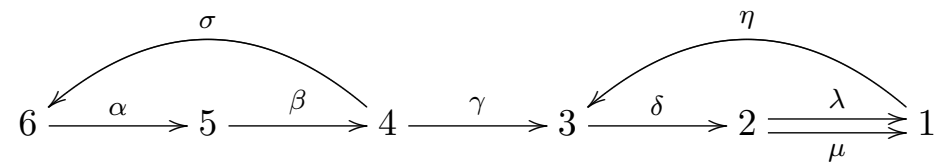

bound by $\alpha \beta=0, \beta \sigma=0, \sigma \alpha=0, \delta \lambda=0, \lambda \eta=0, \eta \delta=0$. This algebra is isomorphic to the relation-extension of the tilted algebra of type $\widetilde{\mathbb{A}}$ given by the quiver

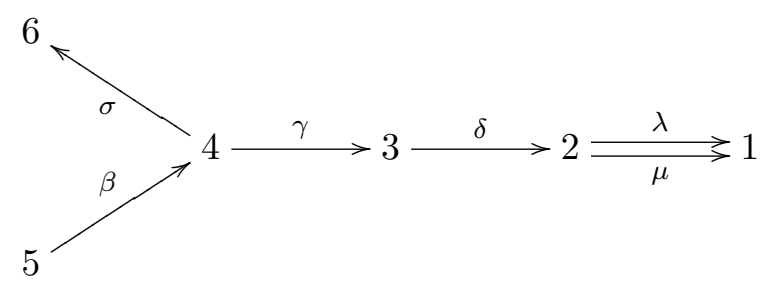

bound by $\beta \sigma=0, \delta \lambda=0$. Therefore $\widetilde{C}$ is cluster-tilted of euclidean type. On the other hand, $C$ is not quasi-tilted, because the uniserial module ${ }_{3}^{4}$ has both projective and injective dimension 2 . Here we represent the module by its Loewy series, see for example [S, 1.5]. 


\section{ReFLECTiOns}

Let $C$ be a tilted algebra. Let $\Sigma$ be a rightmost slice, and let $I(x)$ be an injective source of $\Sigma$. Thus $x$ is a strong sink in $C$.

Definition 4.1. We define the completion $H_{x}$ of $x$ by the following three conditions.

(a) $I(x) \in H_{x}$.

(b) $H_{x}$ is closed under predecessors in $\Sigma$.

(c) If $L \rightarrow M$ is an arrow in $\Sigma$ with $L \in H_{x}$ having an injective successor in $H_{x}$ then $M \in H_{x}$.

Observe that $H_{x}$ may be constructed inductively in the following way. We let $H_{1}=I(x)$, and $H_{2}^{\prime}$ be the closure of $H_{1}$ with respect to (c) (that is, we simply add the direct successors of $I(x)$ in $\Sigma$, and if a direct successor of $I(x)$ is injective, we also take its direct successor, etc.) We then let $H_{2}$ be the closure of $H_{2}^{\prime}$ with respect to predecessors in $\Sigma$. Then we repeat the procedure; given $H_{i}$, we let $H_{i+1}^{\prime}$ be the closure of $H_{i}$ with respect to (c) and $H_{i+1}$ be the closure of $H_{i+1}^{\prime}$ with respect to predecessors. This procedure must stabilize, because the slice $\Sigma$ is finite. If $H_{j}=H_{k}$ with $k>j$, we let $H_{x}=H_{j}$.

We can decompose $H_{x}$ as the disjoint union of three sets as follows. Let $\mathcal{J}$ denote the set of injectives in $H_{x}$, let $\mathcal{J}^{-}$be the set of non-injectives in $H_{x}$ which have an injective successor in $H_{x}$, and let $\mathcal{E}=H_{x} \backslash\left(\mathcal{J} \cup \mathcal{J}^{-}\right)$ denote the complement of $\left(\mathcal{J} \cup \mathcal{J}^{-}\right)$in $H_{x}$. Thus

$$
H_{x}=\mathcal{J} \sqcup \mathcal{J}^{-} \sqcup \mathcal{E}
$$

is a disjoint union.

Remark 4.2. If $\mathcal{J}^{-}=\emptyset$ then $H_{x}$ reduces to the completion $G_{x}$ as defined in [ABS4]. Recall that $G_{x}$ does not always exist, but, as seen above, $H_{x}$ does. Conversely, if $G_{x}$ exists, then it follows from its construction in [ABS4] that $\mathcal{J}^{-}=\emptyset$.

Thus $\mathcal{J}^{-}=\emptyset$ if and only if $G_{x}$ exists, and, in this case $G_{x}=H_{x}$.

For every module $M$ over a cluster-tilted algebra $B$, we can consider a lift $\widetilde{M}$ in the cluster category $\mathcal{C}$. Abusing notation, we sometimes write $\tau^{i} M$ to denote the image of $\tau_{\mathcal{C}}^{i} \widetilde{M}$ in $\bmod B$, and say that the Auslander-Reiten translation is computed in the cluster category.

Definition 4.3. Let $x$ be a strong sink in $C$ and let $\Sigma$ be a rightmost local slice with injective source $I(x)$. Recall that $\Sigma$ is also a local slice in $\bmod B$. Then the reflection of the slice $\Sigma$ in $x$ is

$$
\sigma_{x}^{+} \Sigma=\tau^{-2}\left(\mathcal{J} \cup \mathcal{J}^{-}\right) \cup \tau^{-1} \mathcal{E} \cup\left(\Sigma \backslash H_{x}\right),
$$

where $\tau$ is computed in the cluster category. In a similar way, one defines the coreflection $\sigma_{y}^{-}$of leftmost slices with projective sink $P_{C}(y)$. 
Theorem 4.4. Let $x$ be a strong sink in $C$ and let $\Sigma$ be a rightmost local slice in $\bmod B$ with injective source $I(x)$. Then the reflection $\sigma_{x}^{+} \Sigma$ is a local slice as well.

Proof. Set $\Sigma^{\prime}=\sigma_{x}^{+} \Sigma$ and

$$
\Sigma^{\prime \prime}=\tau^{-1}\left(\mathcal{J} \cup \mathcal{J}^{-}\right) \cup \tau^{-1} \mathcal{E} \cup\left(\Sigma \backslash H_{x}\right)=\tau^{-1} H_{x} \cup\left(\Sigma \backslash H_{x}\right),
$$

where again, $\Sigma^{\prime \prime}$ and $\tau$ are computed in the cluster category $\mathcal{C}$. We claim that $\Sigma^{\prime \prime}$ is a local slice in $\mathcal{C}$. Notice that since $H_{x}$ is closed under predecessors in $\Sigma$, then, if $X \in \Sigma \backslash H_{x}$ is a neighbor of $Y \in H_{x}$, we must have an arrow $Y \rightarrow X$ in $\Sigma$. This observation being made, $\Sigma^{\prime \prime}$ is clearly obtained from $\Sigma$ by applying a sequence of APR-tilts. Thus $\Sigma^{\prime \prime}$ is a local slice in $\mathcal{C}$.

We now claim that $\tau^{-1}\left(\mathcal{J} \cup \mathcal{J}^{-}\right)$is closed under predecessors in $\Sigma^{\prime \prime}$. Indeed, let $X \in \tau^{-1}\left(\mathcal{J} \cup \mathcal{J}^{-}\right)$and $Y \in \Sigma^{\prime \prime}$ be such that we have an arrow $Y \rightarrow X$. Then, there exists an arrow $\tau X \rightarrow Y$ in the cluster category. Because $X \in \tau^{-1}\left(\mathcal{J} \cup \mathcal{J}^{-}\right)$, we have $\tau X \in \mathcal{J} \cup \mathcal{J}^{-}$. Now if $Y \in \Sigma$, then the arrow $\tau X \rightarrow Y$ would imply that $Y \in H_{x}$, which is impossible, because $Y \in \Sigma^{\prime \prime}$ and $\Sigma^{\prime \prime} \cap H_{x}=\emptyset$. Thus $Y \notin \Sigma$, and therefore $Y \in\left(\Sigma^{\prime \prime} \backslash \Sigma\right)=\tau^{-1} H_{x}$. Hence $\tau Y \in H_{x}$. Moreover, there is an arrow $\tau Y \rightarrow \tau X$. Using that $\tau X \in \mathcal{J} \cup \mathcal{J}^{-}$, this implies that $\tau Y$ has an injective successor in $H_{x}$ and thus $Y \in \tau^{-1}\left(\mathcal{J} \cup \mathcal{J}^{-}\right)$. This establishes our claim that $\tau^{-1}\left(\mathcal{J} \cup \mathcal{J}^{-}\right)$is closed under predecessors in $\Sigma^{\prime \prime}$.

Thus applying the same reasoning as before, we get that

$$
\Sigma^{\prime}=\left(\Sigma^{\prime \prime} \backslash \tau^{-1}\left(\mathcal{J} \cup \mathcal{J}^{-}\right)\right) \cup \tau^{-2}\left(\mathcal{J} \cup \mathcal{J}^{-}\right)
$$

is a local slice in $\mathcal{C}$. Now we claim that

$$
\Sigma^{\prime} \cap \operatorname{add}(\tau T)=\emptyset .
$$

First, because $\Sigma \cap \operatorname{add}(\tau T)=\emptyset$, we have $\left(\Sigma \backslash H_{x}\right) \cap \operatorname{add}(\tau T)=\emptyset$. Next, $\mathcal{E}$ contains no injectives, by definition. Thus $\tau^{-1} \mathcal{E} \cap \operatorname{add}(\tau T)=\emptyset$. Assume now that $X \in \operatorname{add}(\tau T)$ belongs to $\tau^{-2} \mathcal{J}^{-}$. Then $\tau^{2} X \in H_{x}$ and there exists an injective predecessor $I(j)$ of $\tau^{2} X$ in $H_{x}$, and since $H_{x}$ is part of the local slice $\Sigma$, there exists a sectional path from $I(j)$ to $\tau^{2} X$. Applying $\tau^{-2}$, we get a sectional path from $T_{j}$ to $X$ in the cluster category. But this means $\operatorname{Hom}_{\mathcal{C}}\left(T_{j}, X\right) \neq 0$, which is a contradiction to the hypothesis that $X \in \operatorname{add}(\tau T)$. Finally, if $X \in \tau^{-2} \mathcal{J}$ then $X$ is a summand of $T$, which, again, is contradicting the hypothesis that $X \in \operatorname{add}(\tau T)$.

Following [ABS4], let $\mathcal{S}_{x}$ be the full subcategory of $C$ consisting of those $y$ such that $I(y) \in H_{x}$.

Lemma 4.5. (a) $\mathcal{S}_{x}$ is hereditary.

(b) $\mathcal{S}_{x}$ is closed under successors in $C$.

(c) $C$ can be written in the form

$$
C=\left[\begin{array}{cc}
H & 0 \\
M & C^{\prime}
\end{array}\right]
$$


where $H$ is hereditary, $C^{\prime}$ is tilted and $M$ is a $C^{\prime}$-H-bimodule.

Proof. (a) Let $H=\operatorname{End}\left(\oplus_{y \in \mathcal{S}_{x}} I(y)\right)$. Then $H$ is a full subcategory of the hereditary endomorphism algebra of $\Sigma$. Therefore $H$ is also hereditary, and so $\mathcal{S}_{x}$ is hereditary.

(b) Let $y \in \mathcal{S}_{x}$ and $y \rightarrow z$ in $C$. Then there exists a morphism $I(z) \rightarrow$ $I(y)$. Because $I(z)$ is an injective $C$-module and $\Sigma$ is sincere, there exist a module $N \in \Sigma$ and a non-zero morphism $N \rightarrow I(z)$. Then we have a path $N \rightarrow I(z) \rightarrow I(y)$, and since $N, I(y) \in \Sigma$, we get that $I(z) \in \Sigma$ by convexity of the slice $\Sigma$ in $\bmod C$. Moreover, since $I(y) \in H_{x}$ and $H_{x}$ is closed under predecessors in $\Sigma$, it follows that $I(z) \in H_{x}$. Thus $z \in \mathcal{S}_{x}$ and this shows (b).

(c) This follows from (a) and (b).

We recall that the cluster duplicated algebra was introduced in [ABS3].

Corollary 4.6. The cluster duplicated algebra $\bar{C}$ of $C$ is of the form

$$
\bar{C}=\left[\begin{array}{cccc}
H & 0 & 0 & 0 \\
M & C^{\prime} & 0 & 0 \\
0 & E_{0} & H & 0 \\
0 & E_{1} & M & C^{\prime}
\end{array}\right]
$$

where $E_{0}=\operatorname{Ext}_{C}^{2}\left(D C^{\prime}, H\right)$ and $E_{1}=\operatorname{Ext}_{C}^{2}\left(D C^{\prime}, C^{\prime}\right)$.

Proof. We start by writing $C$ in the matrix form of the lemma. By definition, $H$ consists of those $y \in C_{0}$ such that the corresponding injective $I(y)$ lies in $H_{x}$ inside the slice $\Sigma$. In particular, the projective dimension of these injectives is at most 1, hence $\operatorname{Ext}_{C}^{2}(D C, C)=\operatorname{Ext}_{C}^{2}\left(D C^{\prime}, C\right)$. The result now follows upon multiplying by idempotents.

Definition 4.7. Let $x$ be a strong sink in $C$. The reflection at $x$ of the algebra $C$ is

$$
\sigma_{x}^{+} C=\left[\begin{array}{cc}
C^{\prime} & 0 \\
E_{0} & H
\end{array}\right]
$$

where $E_{0}=\operatorname{Ext}_{C}^{2}\left(D C^{\prime}, H\right)$.

Proposition 4.8. The reflection $\sigma_{x}^{+} C$ of $C$ is a tilted algebra having $\sigma_{x}^{+} \Sigma$ as a complete slice. Moreover the relation-extensions of $C$ and $\sigma_{x}^{+} \Sigma$ are isomorphic.

Proof. We first claim that the support $\operatorname{supp}\left(\sigma_{x}^{+} \Sigma\right)$ of $\sigma_{x}^{+} \Sigma$ is contained in $\sigma_{x}^{+} C$. Let $X \in \sigma_{x}^{+} \Sigma$. Recall that $\sigma_{x}^{+} \Sigma=\tau^{-2}\left(\mathcal{J} \cup \mathcal{J}^{-}\right) \cup \tau^{-1} \mathcal{E} \cup\left(\Sigma \backslash H_{x}\right)$. If $X \in \tau^{-2} \mathcal{J}$, then $X=P\left(y^{\prime}\right)$ is projective corresponding to a point $y^{\prime} \in H$. Thus $I(y) \in H_{x}$ and the radical of $P(y)$ has no non-zero morphism into $I(y)$. Therefore $\operatorname{supp}(X) \subset \sigma_{X}^{+} C$.

Assume next that $X \in \tau^{-2} \mathcal{J}^{-}$, that is, $X=\tau^{-2} Y$, where $Y \in \mathcal{J}^{-}$has an injective successor $I(z)$ in $H_{x}$. Because all sources in $\Sigma$ are injective, there is an injective $I\left(y^{\prime}\right) \in \Sigma$ and a sectional path $I\left(y^{\prime}\right) \rightarrow \ldots \rightarrow Y \rightarrow \ldots \rightarrow I(z)$. 
Applying $\tau^{-2}$, we obtain a sectional path $P\left(y^{\prime}\right) \rightarrow \ldots \rightarrow X \rightarrow \ldots \rightarrow P(z)$. In particular the point $y^{\prime}$ belongs to the support of $X$. Assume that there is a point $h$ in $H$ that is in the support of $X$. Then there exists a nonzero morphism $X \rightarrow I(h)$. But $I(h) \in \Sigma$ and there is no morphism from $X \in$ $\tau^{-2} \Sigma$ to $\Sigma$. Therefore $\operatorname{supp}(X) \subset \sigma_{x}^{+} C$.

By the same argument, we show that if $X \in \tau^{-1} \mathcal{E}$, then $\operatorname{supp}(X) \subset \sigma_{x}^{+} C$.

Finally, all modules of $\Sigma \backslash H_{x}$ are supported in $C^{\prime}$. This establishes our claim.

Now, by Theorem $4.4, \sigma_{x}^{+} \Sigma$ is a local slice in $\bmod \widetilde{C}$. Therefore $\widetilde{C} / \operatorname{Ann} \sigma_{x}^{+} \Sigma$ is a tilted algebra in which $\sigma_{x}^{+} \Sigma$ is a complete slice. Since the support of $\sigma_{x}^{+} \Sigma$ is the same as the support of $\sigma_{x}^{+} C$, we are done.

We now come to the main result of this section, which states that any two tilted algebras that have the same relation-extension are linked to each other by a sequence of reflections and coreflections.

Definition 4.9. Let $B$ be a cluster-tilted algebra and let $\Sigma$ and $\Sigma^{\prime}$ be two local slices in $\bmod B$. We write $\Sigma \sim \Sigma^{\prime}$ whenever $B / \operatorname{Ann} \Sigma=B / \operatorname{Ann} \Sigma^{\prime}$.

Lemma 4.10. Let $B$ be a cluster-tilted algebra, and $\Sigma_{1}, \Sigma_{2}$ be two local slices in $\bmod B$. Then there exists a sequence of reflections and coreflections $\sigma$ such that

$$
\sigma \Sigma_{1} \sim \Sigma_{2}
$$

Proof. Given a local slice $\Sigma$ in $\bmod B$ such that $\Sigma$ has injective successors in the transjective component $\mathcal{T}$ of $\Gamma(\bmod B)$, let $\Sigma^{+}$be the rightmost local slice such that $\Sigma \sim \Sigma^{+}$. Then $\Sigma^{+}$contains a strong sink $x$, thus reflecting in $x$ we obtain a local slice $\sigma_{x}^{+} \Sigma^{+}$that has fewer injective successors in $\mathcal{T}$ than $\Sigma$. To simplify the notation we define $\sigma_{x}^{+} \Sigma=\sigma_{x}^{+} \Sigma^{+}$. Similarly, we define $\sigma_{y}^{-} \Sigma=\sigma_{y}^{-} \Sigma^{-}$, where $\Sigma^{-}$is the leftmost local slice containing a strong source $y$ and $\Sigma \sim \Sigma^{-}$.

Since we can always reflect in a strong sink, there exist sequences of reflections such that

$$
\begin{gathered}
\sigma_{x_{r}}^{+} \cdots \sigma_{x_{2}}^{+} \sigma_{x_{1}}^{+} \Sigma_{1}=\Sigma_{\infty}^{1} \\
\sigma_{y_{s}}^{+} \cdots \sigma_{y_{2}}^{+} \sigma_{y_{1}}^{+} \Sigma_{2}=\Sigma_{\infty}^{2}
\end{gathered}
$$

and $\Sigma_{\infty}^{1}, \Sigma_{\infty}^{2}$ have no injective successors in $\mathcal{T}$. This implies that $\Sigma_{\infty}^{1} \sim \Sigma_{\infty}^{2}$. Let

thus $\sigma \Sigma_{1} \sim \Sigma_{2}$.

$$
\sigma=\sigma_{y_{1}}^{-} \sigma_{y_{2}}^{-} \cdots \sigma_{y_{s}}^{-} \sigma_{x_{r}}^{+} \cdots \sigma_{x_{2}}^{+} \sigma_{x_{1}}^{+}
$$

Theorem 4.11. Let $C_{1}$ and $C_{2}$ be two tilted algebras that have the same relation-extension. Then there exists a sequence of reflections and coreflections $\sigma$ such that $\sigma C_{1} \cong C_{2}$.

Proof. Let $B$ be the common relation-extension of the tilted algebras $C_{1}$ and $C_{2}$. By [ABS2], there exist local slices $\Sigma_{i}$ in $\bmod B$ such that $C_{i}=$ $B / \operatorname{Ann} \Sigma_{i}$, for $i=1,2$. Now the result follows from Lemma 4.10 and Theorem 4.4 . 
Example 4.12. Let $A$ be the path algebra of the quiver

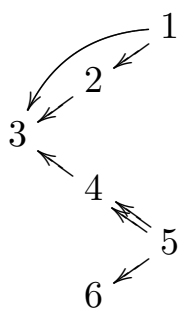

Mutating at the vertices 4,5, and 2 yields the cluster-tilted algebra B with quiver

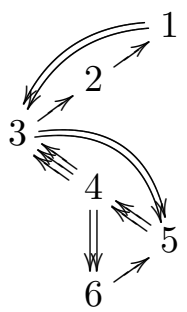

In the Auslander-Reiten quiver of $\bmod B$ we have the following local configuration.

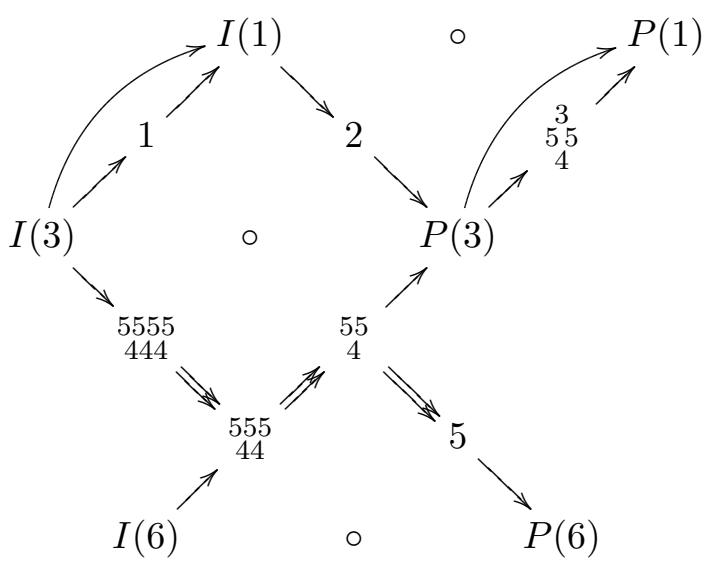

where

$$
I(1)={ }_{1}^{2} \quad I(3)=\underset{3}{2} \underset{3}{2} \underset{444}{5555} \quad I(6)=\underset{6}{555}
$$

The 6 modules on the left form a rightmost local slice $\Sigma$ in which both $I(3)$ and $I(6)$ are sources, so 3 and 6 are strong sinks. For both strong sinks the subset $\mathcal{J}^{-}$of the completion consists of the simple module 1 . The simple module $2=\tau^{-1} 1$ does not lie on a local slice. 
The completion $\mathrm{H}_{6}$ is the whole local slice $\Sigma$ and therefore the reflection $\sigma_{6}^{+} \Sigma$ is the local slice consisting of the 6 modules on the right containing both $P(1)$ and $P(6)$.

On the other hand, the completion $\mathrm{H}_{3}$ consists of the four modules I(3), $S(1), I(1)$ and ${ }_{444}^{555}$, and therefore the reflection $\Sigma^{\prime}=\sigma_{3}^{+} \Sigma$ is the local slice consisting of the 6 modules on the straight line from I(6) to P(1). This local slice admits the strong sink 6 and the completion $H_{6}^{\prime}$ in $\Sigma^{\prime}$ consists of the two modules $I(6)$ and ${ }_{44}^{555}$. Therefore the reflection $\sigma_{6}^{+} \Sigma^{\prime}$ is equal to $\sigma_{6}^{+} \Sigma$. Thus

$$
\sigma_{6}^{+} \Sigma=\sigma_{6}^{+}\left(\sigma_{3}^{+} \Sigma\right)
$$

This example raises the question which indecomposable modules over a cluster-tilted algebra do not lie on a local slice. We answer this question in a forthcoming publication [AsScSe].

\section{TUBES}

The objective of this section is to show how to construct those tubes of a tame cluster-tilted algebra which contain projectives. Let $B$ be a clustertilted algebra of euclidean type, and let $\mathcal{T}$ be a tube in $\Gamma(\bmod B)$ containing at least one projective. First, consider the transjective component of $\Gamma(\bmod B)$. Denote by $\Sigma_{L}$ a local slice in the transjective component that precedes all indecomposable injective $B$-modules lying in the transjective component. Then $B / \operatorname{Ann}_{B} \Sigma_{L}=C_{1}$ is a tilted algebra having a complete slice in the preinjective component. Define $\Sigma_{R}$ to be a local slice which is a successor of all indecomposable projectives lying in the transjective component. Then $B / \operatorname{Ann}_{B} \Sigma_{R}=C_{2}$ is a tilted algebra having a complete slice in the postprojective component. Also, $C_{1}$ (respectively, $C_{2}$ ) has a tube $\mathcal{T}_{1}$ (respectively, $\mathcal{T}_{2}$ ) containing the indecomposable projective $C_{1}$-modules (respectively, injective $C_{2}$-modules) corresponding to the projective $B$-modules in $\mathcal{T}$ (respectively, injective $B$-modules in $\mathcal{T}$ ).

An indecomposable projective $P(x)$ (respectively, injective $I(x)) B$-module that lies in a tube, is said to be a root projective (respectively, a root injective) if there exists an arrow in $B$ between $x$ and $y$, where the corresponding indecomposable projective $P(y)$ lies in the transjective component of $\Gamma(\bmod B)$.

Let $\mathcal{S}_{1}$ be the coray in $\mathcal{T}_{1}$ passing through the projective $C_{1}$-module that corresponds to the root projective $P_{B}(i)$ in $\mathcal{T}$. Similarly, let $\mathcal{S}_{2}$ be the ray in $\mathcal{T}_{2}$ passing through the injective that corresponds to the root injective $I_{B}(i)$ in $\mathcal{T}$.

Recall that if $A$ is hereditary and $T \in \bmod A$ is a tilting module, then there exists an associated torsion pair $(\mathscr{T}(T), \mathscr{F}(T))$ in $\bmod A$, where

$$
\begin{gathered}
\mathscr{T}(T)=\left\{M \in \bmod A \mid \operatorname{Ext}_{A}^{1}(T, M)=0\right\} \\
\mathscr{F}(T)=\left\{M \in \bmod A \mid \operatorname{Hom}_{A}(T, M)=0\right\},
\end{gathered}
$$


see for example [ASS, VI.2]

Lemma 5.1. With the above notation

(a) $\mathcal{S}_{1} \otimes_{C_{1}} B$ is a coray in $\mathcal{T}$ passing through $P_{B}(i)$.

(b) $\operatorname{Hom}_{C_{2}}\left(B, \mathcal{S}_{2}\right)$ is a ray in $\mathcal{T}$ passing through $I_{B}(i)$.

Proof. Since $C_{1}$ is tilted, we have $C_{1}=\operatorname{End}_{A} T$ where $T$ is a tilting module over a hereditary algebra $A$. As seen in the proof of Theorem 5.1 in [ScSe], we have a commutative diagram

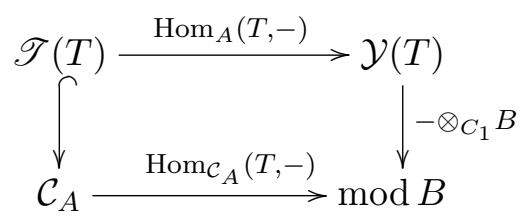

where $\mathcal{Y}(T)=\left\{N \in \bmod C \mid \operatorname{Tor}_{1}^{C}(N, T)=0\right\}$.

Let $\mathcal{T}_{A}$ be the tube in $\bmod A$ corresponding to the tube $\mathcal{T}$ in $\bmod B$. By what has been seen above, we have a commutative diagram

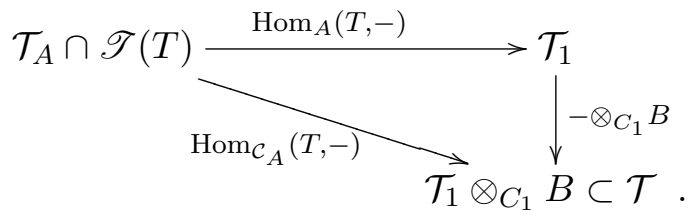

Let $\mathcal{S}$ be any coray in $\mathcal{T}_{1}$, so it can be lifted to a coray $\mathcal{S}_{A}$ in $\mathcal{T}_{A} \cap \mathscr{T}(T)$ via the functor $\operatorname{Hom}_{A}(T,-)$. If we apply $\operatorname{Hom}_{\mathcal{C}_{A}}(T,-)$ to this lift, we obtain a coray in $\mathcal{T}_{1} \otimes_{C_{1}} B$. Thus, any coray in $\mathcal{T}_{1}$ induces a coray in $\mathcal{T}$. Let $\mathcal{S}_{1}$ be the coray passing through the root projective $P_{C_{1}}(i)$. Then $\mathcal{S}_{1} \otimes_{C_{1}} B$ is the coray passing through $P_{C_{1}}(i) \otimes_{C_{1}} B=P_{B}(i)$. This proves (a) and part (b) is proved dually.

However, we must still justify that the ray $\mathcal{S}_{1} \otimes_{C_{1}} B$ and the coray $\operatorname{Hom}_{\mathcal{C}_{2}}\left(B, \mathcal{S}_{2}\right)$ actually intersect (and thus lie in the same tube of $\left.\Gamma(\bmod B)\right)$. Because $P_{C_{1}}(i) \in \mathcal{S}_{1}$, we have $P_{C_{1}}(i) \otimes B \cong P_{B}(i) \in \mathcal{S}_{1} \otimes_{C_{1}} B$, and $P_{B}(i)$ lies in a tube $\mathcal{T}$. It is well-known that the injective $I_{B}(i)$ also lies in $\mathcal{T}$. In particular, we have the following local configuration in $\mathcal{T}$, where $R$ is an indecomposable summand of the radical of $P_{B}(i)$ and $J$ an indecomposable summand of the quotient of $I_{B}(i)$ by its socle.

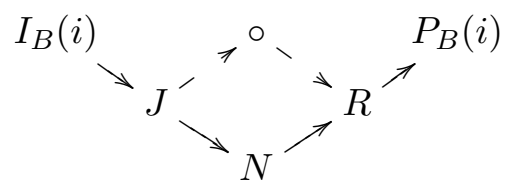

Now $I_{B}(i)=\operatorname{Hom}_{C_{2}}\left(B, I_{C}(i)\right)$ is coinduced, and we have shown above that the ray containing it is also coinduced. Because $I_{C}(i) \in \mathcal{S}_{2}$, this is the ray $\operatorname{Hom}_{C_{2}}\left(B, \mathcal{S}_{2}\right)$. Therefore, this ray and this coray lie in the same tube, so must intersect in a module $N$, where there exists an almost split sequence 
$0 \longrightarrow J \longrightarrow N \longrightarrow R \longrightarrow 0$

Remark 5.2. Knowing the ray $\operatorname{Hom}_{C_{2}}\left(B, \mathcal{S}_{2}\right)$ and the coray $\mathcal{S}_{1} \otimes_{C_{1}} B$ for every root projective $P_{B}(i)$ in $\mathcal{T}$, one may apply the knitting procedure (see for example [S, Chapter 3]) to construct the whole of $\mathcal{T}$. In this way, $\mathcal{T}$ can be determined completely.

Next we show that all modules over a tilted algebra lying on the same coray change in the same way under the induction functor.

Lemma 5.3. Let $A$ be a hereditary algebra of euclidean type, $T$ a tilting $A$-module without preinjective summands and let $C=\operatorname{End}_{A} T$ be the corresponding tilted algebra. Let $\mathcal{T}_{A}$ be a tube in $\bmod A$ and $T_{i} \in \mathcal{T}_{A}$ an indecomposable summand of $T$, such that $\mathrm{pd} I_{C}(i)=2$.

Then there exists an $A$-module $M$ on the mouth of $\mathcal{T}_{A}$ such that we have

$$
\tau_{C} \Omega_{C} I_{C}(i)=\operatorname{Hom}_{A}(T, M)
$$

in $\bmod C$. In particular, the module $\tau_{C} \Omega_{C} I_{C}(i)$ lies on the mouth of the tube $\operatorname{Hom}_{A}\left(T, \mathcal{T}_{A} \cap \mathscr{T}(T)\right)$ in $\bmod C$.

Proof. The injective $C$-module $I_{C}(i)$ is given by

$$
I_{C}(i) \cong \operatorname{Ext}_{A}^{1}\left(T, \tau T_{i}\right) \cong D \operatorname{Hom}_{A}\left(T_{i}, T\right)
$$

where the first identity holds by [ASS, Proposition VI 5.8] and the second identity is the Auslander-Reiten formula. Moreover, since $T_{i}$ lies in the tube $\mathcal{T}_{A}$ and $T$ has no preinjective summands, we have $\operatorname{Hom}\left(T_{i}, T_{j}\right) \neq 0$ only if $T_{j}$ lies in the hammock starting at $T_{i}$. Furthermore, if $T_{j}$ is a summand of $T$ then it must lie on a sectional path starting from $T_{i}$ because $\operatorname{Ext}^{1}\left(T_{j}, T_{i}\right)=0$. This shows that a point $j$ is in the support of $I_{C}(i)$ if and only if there is a sectional path $T_{i} \rightarrow \cdots \rightarrow T_{j}$ in $\mathcal{T}_{A}$. We shall distinguish two cases.

Case 1. If $T_{i}$ lies on the mouth of $\mathcal{T}_{A}$ then let $\omega$ be the ray starting at $T_{i}$ and denote by $T_{1}$ the last summand of $T$ on this ray. Let $L_{1}$ be the direct predecessor of $T_{1}$ not on the ray $\omega$. Thus we have the following local configuration in $\mathcal{T}_{A}$. 


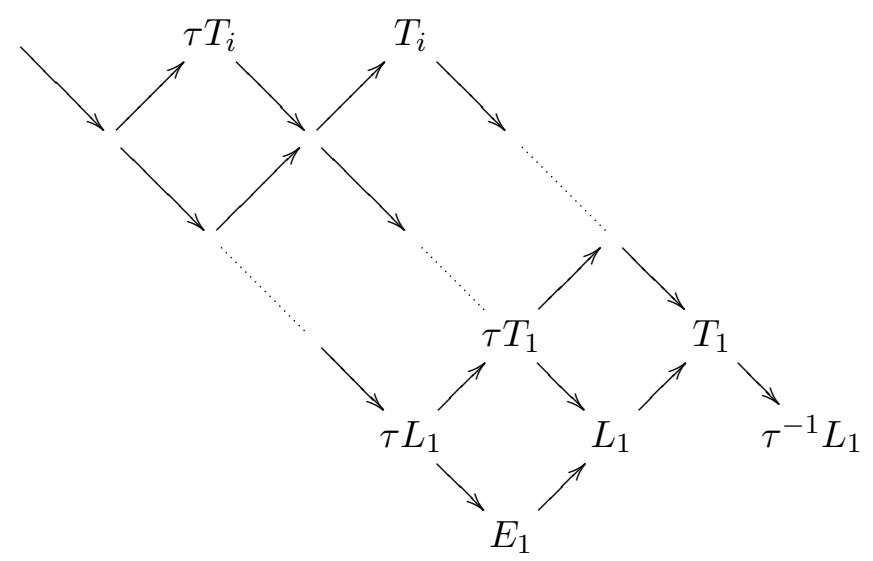

Then $I_{C}(i)$ is uniserial with simple top $S(1)$. Moreover there is a short exact sequence

$$
0 \longrightarrow \tau T_{i} \longrightarrow L_{1} \longrightarrow T_{1} \longrightarrow 0
$$

and applying $\operatorname{Hom}_{A}(T,-)$ yields

$$
0 \longrightarrow \operatorname{Hom}_{A}\left(T, L_{1}\right) \longrightarrow P_{C}(1) \stackrel{f}{\longrightarrow} I_{C}(i) \longrightarrow \operatorname{Ext}^{1}\left(T, L_{1}\right) \longrightarrow 0
$$

By the Auslander-Reiten formula, we have $\operatorname{Ext}^{1}\left(T, L_{1}\right) \cong D \operatorname{Hom}\left(\tau^{-1} L_{1}, T\right)$ and this is zero because $T_{1}$ is the last summand of $T$ on the ray $\omega$. Thus the sequence (5.1) is short exact, the morphism $f$ is a projective cover, because $I_{C}(i)$ is uniserial, and hence

$$
\Omega_{C} I_{C}(i) \cong \operatorname{Hom}_{A}\left(T, L_{1}\right)
$$

Applying $\tau_{C}$ yields

$$
\tau_{C} \Omega_{C} I_{C}(i) \cong \tau_{C} \operatorname{Hom}_{A}\left(T, L_{1}\right)
$$

Let $E_{1}$ be the indecomposable direct predecessor of $L_{1}$ such that the almost split sequence ending at $L_{1}$ is of the form

$$
0 \longrightarrow \tau L_{1} \longrightarrow E_{1} \oplus \tau T_{1} \longrightarrow L_{1} \longrightarrow 0
$$

We claim that $E_{1} \in \mathscr{T}(T)$.

Recall that $L_{1}$ is not a summand of $T$ because $\Omega_{C} I_{C}(i)=\operatorname{Hom}_{A}\left(T, L_{1}\right)$ is non projective. Also, recall that $T_{1}$ is the last summand of $T$ on the ray $\omega$. Suppose $E_{1} \notin \mathscr{T}(T)$, thus $0 \neq \operatorname{Ext}_{A}^{1}\left(T, E_{1}\right)=D \operatorname{Hom}\left(\tau^{-1} E_{1}, T\right)$. Then it follows that there is a summand of $T$ on the ray $\tau \omega$ that is a successor of $\tau^{-1} E_{1}$. Let $T^{1}$ denote the first such indecomposable summand. 


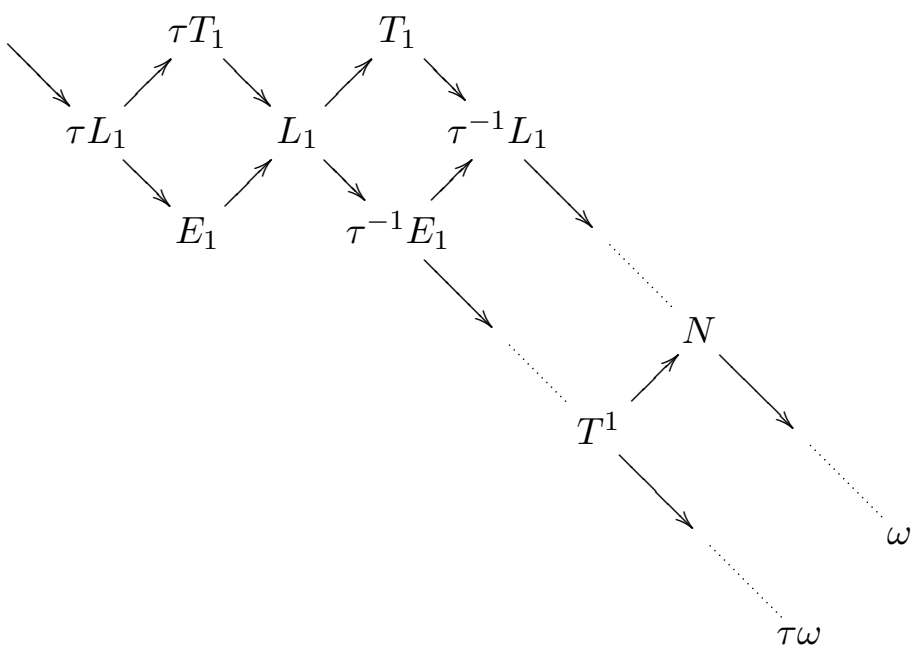

Then we have a short exact sequence

$$
0 \longrightarrow L_{1} \stackrel{h}{\longrightarrow} T_{1} \oplus T^{1} \longrightarrow N \longrightarrow 0
$$

with $h$ an add $T$-approximation. Applying $\operatorname{Hom}_{A}(-, T)$ yields

$$
\begin{aligned}
& 0 \longrightarrow \operatorname{Hom}_{A}(N, T) \longrightarrow \operatorname{Hom}_{A}\left(T_{1} \oplus T^{1}, T\right) \stackrel{h^{*}}{\longrightarrow} \operatorname{Hom}_{A}\left(L_{1}, T\right) \\
& \longrightarrow \operatorname{Ext}_{A}^{1}(N, T) \longrightarrow 0
\end{aligned}
$$

and since $h$ is an add $T$-approximation, the morphism $h^{*}$ is surjective. Thus $\operatorname{Ext}_{A}^{1}(N, T)=0$.

On the other hand, $T_{1} \oplus T^{1}$ generates $N$, so $N \in \operatorname{Gen} T=\mathscr{T}(T)$, and thus $\operatorname{Ext}_{A}^{1}(T, N)=0$. But then both $\operatorname{Ext}_{A}^{1}(T, N)=\operatorname{Ext}_{A}^{1}(N, T)=0$ and we see that $N$ is a summand of $T$. This is a contradiction to the assumption that $T_{1}$ is the last summand of $T$ on the ray $\omega$. Thus $E_{1} \in \mathscr{T}(T)$.

Therefore, in the almost split sequence (5.2), we have $L_{1}, E_{1} \in \mathscr{T}(T)$ and $\tau T_{1} \in \mathscr{F}(T)$. Moreover, all predecessors of $\tau T_{1}$ on the ray $\tau \omega$ are also in $\mathscr{F}(T)$ because the morphisms on the ray are injective. Since $\operatorname{Hom}_{A}(T,-)$ : $\mathscr{T}(T) \rightarrow \mathcal{Y}(T)$ is an equivalence of categories, it follows that $\operatorname{Hom}_{A}\left(T, L_{1}\right)$ has only one direct predecessor

$$
\operatorname{Hom}_{A}\left(T, E_{1}\right) \rightarrow \operatorname{Hom}_{A}\left(T, L_{1}\right)
$$

in $\bmod C$ and this irreducible morphism is surjective. The kernel of this morphism is $\operatorname{Hom}_{A}\left(T, t\left(\tau_{A} L_{1}\right)\right)$ where $t$ is the torsion radical. Thus we get

$$
\tau_{C} \Omega_{C} I_{C}(i)=\tau_{C} \operatorname{Hom}_{A}\left(T, L_{1}\right)=\operatorname{Hom}_{A}\left(T, t\left(\tau_{A} L_{1}\right)\right) .
$$

We will show that $t\left(\tau_{A} L_{1}\right)$ lies on the mouth of $\mathcal{T}_{A}$ and this will complete the proof in case 1 . 
Let $M$ be the indecomposable $A$-module on the mouth of $\mathcal{T}_{A}$ such that the ray starting at $M$ passes through $\tau_{A} L_{1}$. Thus $M$ is the starting point of the ray $\tau^{2} \omega$. Then there is a short exact sequence of the form

$$
0 \longrightarrow M \longrightarrow \tau_{A} L_{1} \longrightarrow \tau_{A} T_{1} \longrightarrow 0
$$

with $\tau_{A} T_{1} \in \mathscr{F}(T)$. We claim that $M \in \mathscr{T}(T)$.

Suppose to the contrary that $0 \neq \operatorname{Ext}_{A}^{1}(T, M)=D \operatorname{Hom}_{A}\left(\tau^{-1} M, T\right)$. Since $\tau^{-1} M$ lies on the mouth of $\mathcal{T}_{A}$, this implies that there is a direct summand $T^{1}$ of $T$ which lies on the ray $\tau \omega$ starting at $\tau^{-1} M$. Since $T$ is tilting, $T^{1}$ cannot be a predecessor of $\tau T_{1}$ on this ray and since $L_{1}$ is not a summand of $T$, we also have $L_{1} \neq T^{1}$. Thus $T^{1}$ is a successor of $L_{1}$ on the ray $\tau \omega$. This is impossible since such a $T^{1}$ would satisfy $\operatorname{Ext}_{A}^{1}\left(T^{1}, E_{1}\right) \neq 0$ contradicting the fact that $E_{1} \in \mathscr{T}(T)$.

Therefore, $M \in \mathscr{T}(T)$ and the sequence (5.3) is the canonical sequence for $\tau_{A} L_{1}$ in the torsion pair $(\mathscr{T}(T), \mathscr{F}(T))$. In other words, $M$ is the largest submodule of $\tau_{A} L_{1}$ in $\mathscr{T}(T)$ and the quotient $\tau_{A} T_{1}$ lies in $\mathscr{F}(T)$, see [ASS, VI.1]. This shows that $t\left(\tau_{A} L_{1}\right)=M$ and hence $\tau_{C} \Omega_{C} I_{C}(i)=\operatorname{Hom}_{A}(T, M)$ as desired.

Case 2. Now suppose that $T_{i}$ does not lie on the mouth of $\mathcal{T}_{A}$. Let $\omega_{1}$ denote the ray passing through $T_{i}$ and $\omega_{2}$ the coray passing through $T_{i}$. Denote by $T_{1}$ the last summand of $T$ on $\omega_{1}$, by $T_{2}$ the last summand of $T$ on $\omega_{2}$, and by $L_{j}$ the direct predecessor of $T_{j}$ which does not lie on $\omega_{j}$. Note that $L_{2}$ does not exist if $T_{2}$ lies on the mouth of $\mathcal{T}_{A}$, and in this case we let $L_{2}=0$. Thus we have the following local configuration in $\mathcal{T}_{A}$.

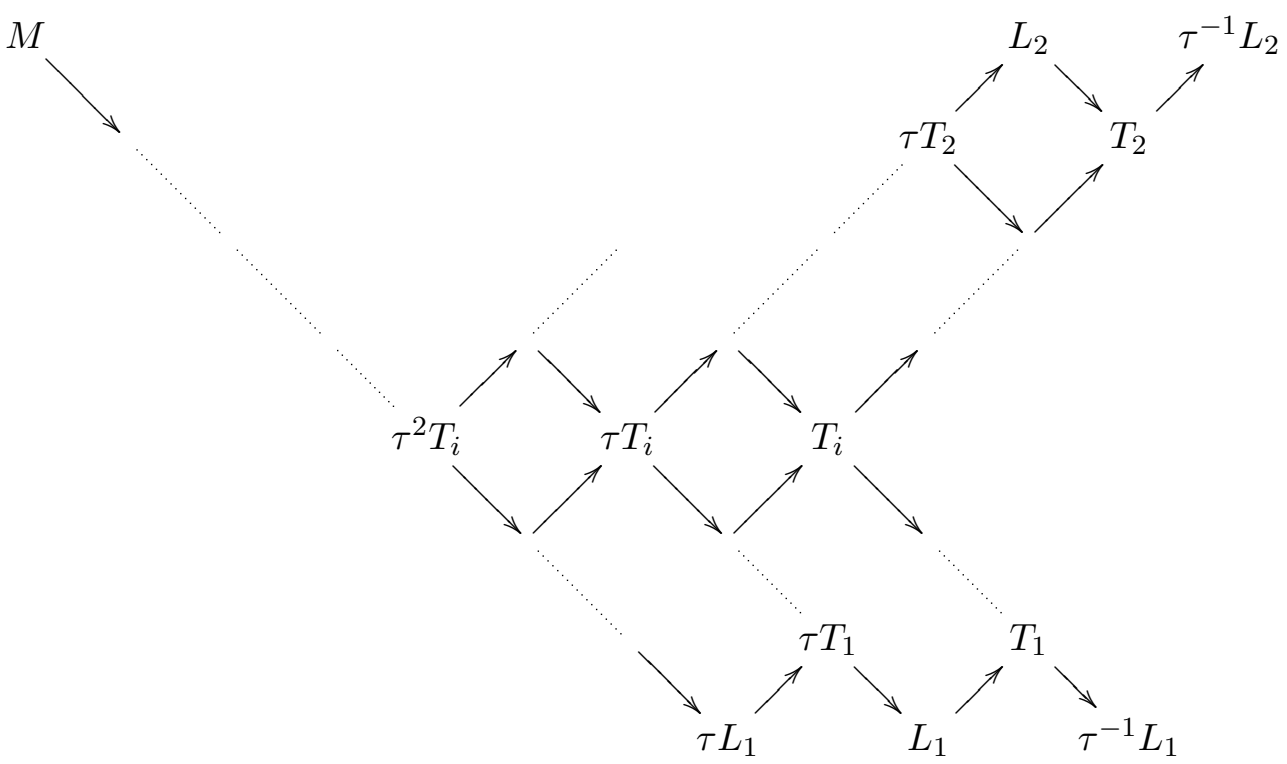


The injective $C$-module $I_{C}(i)=\operatorname{Ext}_{A}^{1}\left(T, \tau T_{i}\right)$ is biserial with top $S(1) \oplus$ $S(2)$. Moreover, there is a short exact sequence

$$
0 \longrightarrow \tau T_{i} \longrightarrow L_{1} \oplus L_{2} \oplus T_{i} \longrightarrow T_{1} \oplus T_{2} \longrightarrow 0 .
$$

Applying $\operatorname{Hom}_{A}(T,-)$ yields the following exact sequence.

$$
\begin{aligned}
0 \longrightarrow \operatorname{Hom}_{A}\left(T, L_{1} \oplus L_{2}\right) \oplus P_{C}(i) \longrightarrow P_{C}(1) \oplus P_{C}(2) \stackrel{f}{\longrightarrow} I_{C}(i) \\
\longrightarrow \operatorname{Ext}_{A}^{1}\left(T, L_{1} \oplus L_{2}\right) \longrightarrow
\end{aligned}
$$

By the same argument as in case 1 , using that $T_{1}$ and $T_{2}$ are the last summands of $T$ on $\omega_{1}$ and $\omega_{2}$ respectively, we see that $\operatorname{Ext}_{A}^{1}\left(T, L_{1} \oplus L_{2}\right)=0$. Therefore, the sequence (5.4) is short exact. Moreover, the morphism $f$ is a projective cover and thus

$$
\Omega_{C} I_{C}(i)=\operatorname{Hom}_{A}\left(T, L_{1} \oplus L_{2}\right) \oplus P_{C}(i) .
$$

Applying $\tau_{C}$ yields

$$
\tau_{C} \Omega_{C} I_{C}(i)=\tau_{C} \operatorname{Hom}_{A}\left(T, L_{1}\right) \oplus \tau_{C} \operatorname{Hom}_{A}\left(T, L_{2}\right) .
$$

By the same argument as in case 1 we see that

$$
\tau_{C} \operatorname{Hom}_{A}\left(T, L_{1}\right)=\operatorname{Hom}_{A}\left(T, t\left(\tau_{A} L_{1}\right)\right)=\operatorname{Hom}_{A}(T, M)
$$

where $M$ is the indecomposable $A$-module on the mouth of $\mathcal{T}_{A}$ such that the ray starting at $M$ passes through $\tau L_{1}$. In other words, $M$ is the starting point of the ray $\tau^{2} \omega$.

Therefore, it only remains to show that $\tau_{C} \operatorname{Hom}_{A}\left(T, L_{2}\right)=0$. To do so, it suffices to show that $L_{2}$ is a summand of $T$.

We have already seen that $\operatorname{Ext}_{A}^{1}\left(T, L_{2}\right)=0$. We show now that we also have $\operatorname{Ext}_{A}^{1}\left(L_{2}, T\right)=0$. Suppose the contrary. Then there exists a nonzero morphism $u: T \rightarrow \tau_{A} L_{2}$. Composing it with the irreducible injective morphism $\tau_{A} L_{2} \rightarrow \tau_{A} T_{2}$ yields a non-zero morphism in $\operatorname{Hom}_{A}\left(T, \tau_{A} T_{2}\right)$. But this is impossible since $T$ is tilting.

Thus we have $\operatorname{Ext}_{A}^{1}\left(T, L_{2}\right)=\operatorname{Ext}_{A}^{1}\left(L_{2}, T\right)=0$ and thus $L_{2}$ is a summand of $T$, the module $\operatorname{Hom}_{A}\left(T, L_{2}\right)$ is projective and $\tau_{C} \operatorname{Hom}_{A}\left(T, L_{2}\right)=0$. This completes the proof.

Remark 5.4. The module $M$ in the statement of the lemma is the starting point of the ray passing through $\tau^{2} T_{i}$.

Corollary 5.5. Let $A, T, C, \mathcal{T}_{A}$ be as in Lemma 5.3, and let $B=C \ltimes E$, with $E=\operatorname{Ext}_{C}^{2}(D C, C)$. Let $X, Y$ be two modules lying on the same coray in the tube $\operatorname{Hom}_{A}\left(T, \mathcal{T}_{A} \cap \mathscr{T}(T)\right)$ in $\bmod C$. Then $X \otimes_{C} E \cong Y \otimes_{C} E$ and thus the two projections $X \otimes_{C} B \rightarrow X \rightarrow 0$ and $Y \otimes_{C} B \rightarrow Y \rightarrow 0$ have isomorphic kernels. 
Proof. For all $C$-modules $X$ we have

$$
X \otimes_{B} E \cong D \operatorname{Hom}(X, D E) \cong D \operatorname{Hom}\left(X, \tau_{C} \Omega_{C} D C\right)
$$

where the first isomorphism is [ScSe, Proposition 3.3] and the second is [ScSe, Proposition 4.1]. Since $T$ has no preinjective summands, and $X$ is regular, the only summand of $\tau \Omega D C$ for which $\operatorname{Hom}(X, \tau \Omega D C)$ can be nonzero, must lie in the same tube as $X$. By the lemma, the only summands of $\tau \Omega D C$ in the tube lie on the mouth of the tube. Let $M$ denote an indecomposable $C$-module on the mouth of a tube. Then

$$
\operatorname{Hom}_{C}(X, M) \cong \operatorname{Hom}_{C}(Y, M) \cong\left\{\begin{array}{cc}
k & \text { if } M \text { lies on the coray passing } \\
& \text { through } X \text { and } Y \\
0 & \text { otherwise }
\end{array}\right.
$$

We summarize the results of this section in the following proposition.

Proposition 5.6. $\quad$ (a) Let $\mathcal{S}_{1}$ be the coray in $\Gamma\left(\bmod C_{1}\right)$ passing through the projective $C_{1}$-module corresponding to the root projective $P_{B}(i)$ Then $\mathcal{S}_{1} \otimes_{C_{1}} B$ is a coray in $\Gamma(\bmod B)$ passing through $P_{B}(i)$. Furthermore all modules in $\mathcal{S}_{1} \otimes_{C_{1}} B$ are extensions of modules of $\mathcal{S}_{1}$ by the same module $P_{C_{1}}(i) \otimes E$.

(b) Let $\mathcal{S}_{2}$ be the ray in $\Gamma\left(\bmod C_{2}\right)$ passing through the injective $C_{2}$ module corresponding to the root injective $I_{B}(i)$ Then $\operatorname{Hom}_{C_{2}}\left(B, \mathcal{S}_{2}\right)$ is a ray in $\Gamma(\bmod B)$ passing through $I_{B}(i)$. Furthermore all modules in $\operatorname{Hom}_{C_{2}}\left(B, \mathcal{S}_{2}\right)$ are extensions of modules of $\mathcal{S}_{2}$ by the same module $\operatorname{Hom}_{C_{2}}\left(E, I_{C_{2}}(i)\right)$.

Proof. (a) The first statement is Lemma 5.1, and the second statement is a restatement of Corollary 5.5.

Example 5.7. Let $B$ be the cluster-tilted algebra given by the quiver

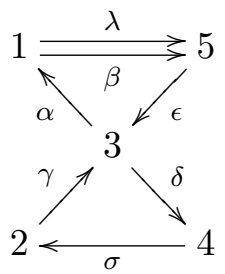

bound by $\alpha \beta=0, \beta \epsilon=0, \epsilon \alpha=0, \gamma \delta=0, \sigma \gamma=0, \delta \sigma=0$. The algebras $C_{1}$ and $C_{2}$ are respectively given by the quivers 

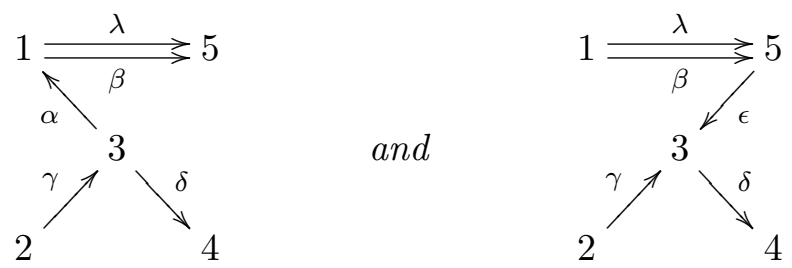

with the inherited relations. We can see the tube in $\Gamma\left(\bmod C_{1}\right)$ below and the coray passing through the root projective $P_{C_{1}}(3)={ }_{4}^{3} \frac{1}{5}$ is given by

$$
\mathcal{S}_{1}: \quad \ldots \longrightarrow \frac{1}{5} \longrightarrow 4^{3} \frac{1}{5} \longrightarrow \begin{aligned}
& 3 \\
& 1
\end{aligned} \longrightarrow \begin{aligned}
& 2 \\
& 5 \\
& \frac{1}{5}
\end{aligned}
$$

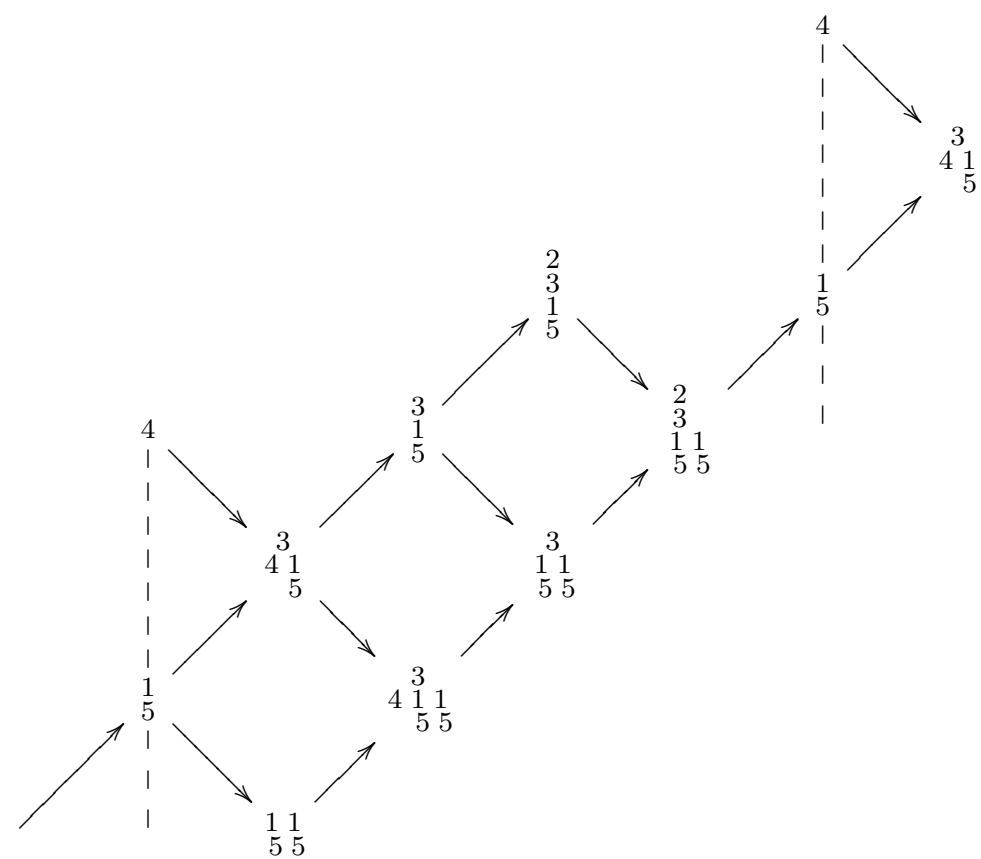

Dually, the ray in $\Gamma\left(\bmod C_{2}\right)$ passing through the root injective $I_{C_{2}}(3)=$ ${ }_{5}^{1} 2$ is given by

$$
\mathcal{S}_{2}: \quad \begin{aligned}
& 1 \\
& 5 \\
& 3
\end{aligned} \quad \begin{aligned}
& 1 \\
& 4
\end{aligned}{ }_{3}^{5} \longrightarrow 5_{3}^{1} \longrightarrow{ }_{5} \longrightarrow \ldots
$$

The root projective $P_{B}(3)$ lies on the coray

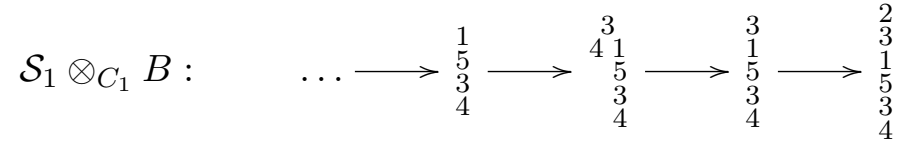

and the root injective $I_{B}(3)$ lies on the ray 


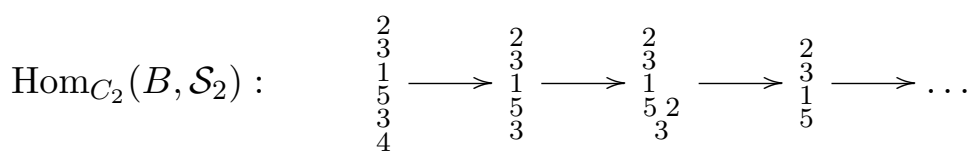

Note that by Proposition 5.6, every module in $\mathcal{S}_{1} \otimes_{C_{1}} B$ is an extension of a module in $\mathcal{S}_{1}$ by ${ }_{4}^{3}$. Similarly, every module in $\operatorname{Hom}_{C_{2}}\left(B, \mathcal{S}_{2}\right)$ is an extension of a module in $\mathcal{S}_{2}$ by ${ }_{3}^{2}$.

Applying the knitting algorithm we obtain the tube in $\Gamma(\bmod B)$ containing both $\mathcal{S}_{1} \otimes_{C_{1}} B$ and $\operatorname{Hom}_{C_{2}}\left(B, \mathcal{S}_{2}\right)$.

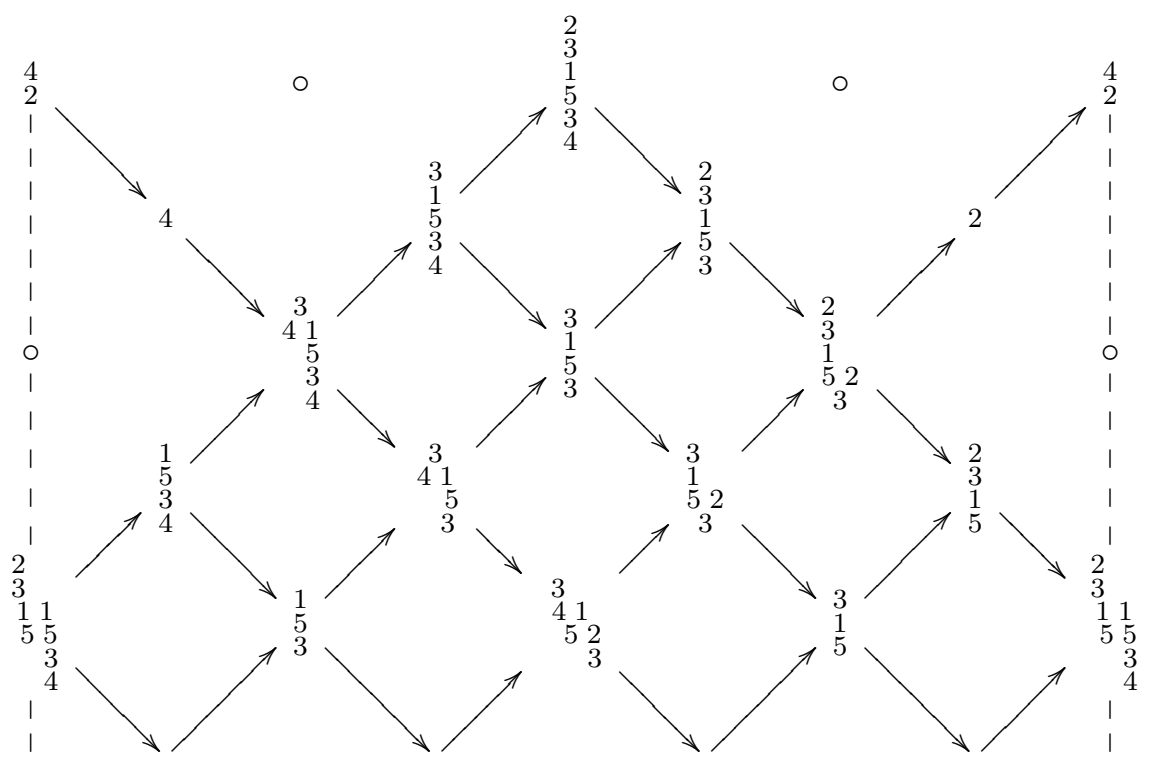

\section{From Cluster-tilted Algebras to QUASI-TILTED ALGEBras}

Let $B$ be cluster-tilted of euclidean type $Q$ and let $A=k Q$. Then there exists $T \in \mathcal{C}_{A}$ tilting such that $B=\operatorname{End}_{\mathcal{C}_{A}} T$.

Because $Q$ is euclidean, $\mathcal{C}_{A}$ contains at most 3 exceptional tubes. Denote by $T_{0}, T_{1}, T_{2}, T_{3}$ the direct sums of those summands of $T$ that respectively lie in the transjective component and in the three exceptional tubes.

In the derived category $\mathcal{D}^{b}(\bmod A)$, we can choose a lift of $T$ such that we have the following local configuration.

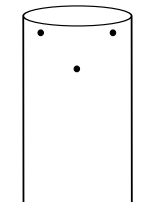

$T_{1}$

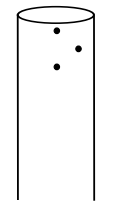

$T_{2}$

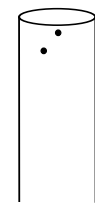

$T_{3}$

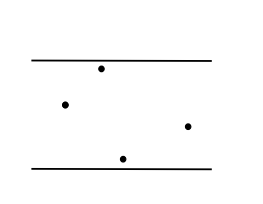

$T_{0}$

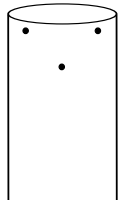

$F T_{1}$

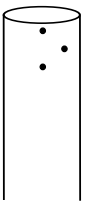

$F T_{2}$

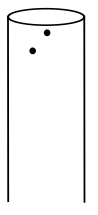

$F T_{3}$

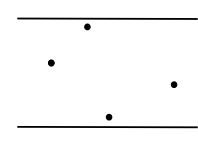

$F T_{0}$ 
Let $\mathcal{H}$ be a hereditary category that is derived equivalent to $\bmod A$ and such that $\mathcal{H}$ is not the module category of a hereditary algebra. Then $\mathcal{H}$ is of the form $\mathcal{H}=\mathcal{T}^{-} \vee \mathcal{C} \vee \mathcal{T}^{+}$, where $\mathcal{T}^{-}, \mathcal{T}^{+}$consist of tubes, and $\mathcal{C}$ is a transjective component, see Proposition 2.2 and its subsequent remarks in [LS]. Let $T_{-}, T_{+}$be the direct sum of all indecomposable summands of $T$ lying in $\mathcal{T}^{-}, \mathcal{T}^{+}$respectively. We define two subspaces $L$ and $R$ of $B$ as follows.

$$
L=\operatorname{Hom}_{\mathcal{D}^{b}(\bmod A)}\left(F^{-1} T_{+}, T_{0}\right) \quad \text { and } \quad R=\operatorname{Hom}_{\mathcal{D}^{b}(\bmod A)}\left(T_{0}, F T_{-}\right) .
$$

The transjective component of $\bmod B$ contains a left section $\Sigma_{L}$ and a right section $\Sigma_{R}$, see [A, Section 2]. Thus $\Sigma_{L}, \Sigma_{R}$ are local slices, $\Sigma_{L}$ has no projective predecessors, and $\Sigma_{R}$ has no projective successors in the transjective component. Define $K$ to be the two-sided ideal of $B$ generated by $\operatorname{Ann} \Sigma_{L} \cap \operatorname{Ann} \Sigma_{R}$ and the two subspaces $L$ and $R$. Thus

$$
K=\left\langle\operatorname{Ann} \Sigma_{L} \cap \operatorname{Ann} \Sigma_{R}, L, R\right\rangle .
$$

We call $K$ the partition ideal induced by the partition $\mathcal{T}^{-} \vee \mathcal{C} \vee \mathcal{T}^{+}$.

Theorem 6.1. The algebra $C=B / K$ is quasi-tilted and such that $B=\widetilde{C}$. Moreover $C$ is tilted if and only if $L=0$ or $R=0$.

Proof. We have $B=\operatorname{End}_{\mathcal{C}_{A}} T=\oplus_{i \in \mathbb{Z}} \operatorname{Hom}_{\mathcal{D}^{b}(\bmod A)}\left(T, F^{i} T\right)$, where the last equality is as $k$-vector spaces. Using the decomposition $T=T_{-} \oplus T_{0} \oplus T_{+}$, we see that $B$ is equal to

$$
\begin{array}{cccccc} 
& \operatorname{Hom}_{\mathcal{D}}\left(T_{-}, T_{-}\right) & \oplus & \operatorname{Hom}_{\mathcal{D}}\left(T_{-}, T_{0}\right) & \oplus & \operatorname{Hom}_{\mathcal{D}}\left(T_{-}, F T_{-}\right) \\
\oplus & \operatorname{Hom}_{\mathcal{D}}\left(T_{0}, T_{0}\right) & \oplus & \operatorname{Hom}_{\mathcal{D}}\left(T_{0}, T_{+}\right) & \oplus & \operatorname{Hom}_{\mathcal{D}}\left(T_{0}, F T_{-}\right) \\
\oplus & \operatorname{Hom}_{\mathcal{D}}\left(T_{0}, F T_{0}\right) & \oplus & \operatorname{Hom}_{\mathcal{D}}\left(F^{-1} T_{+}, F T_{0}\right) & \oplus & \operatorname{Hom}_{\mathcal{D}}\left(F^{-1} T_{+}, T_{+}\right) \\
\oplus & \operatorname{Hom}_{\mathcal{D}}\left(T_{+}, T_{+}\right), & & & &
\end{array}
$$

where all Hom spaces are taken in $\mathcal{D}^{b}(\bmod A)$. On the other hand,

$$
\begin{aligned}
\operatorname{End}_{\mathcal{H}} T & =\operatorname{Hom}_{\mathcal{H}}\left(T_{-}, T_{-}\right) \oplus \operatorname{Hom}_{\mathcal{H}}\left(T_{-}, T_{0}\right) \oplus \operatorname{Hom}_{\mathcal{H}}\left(T_{0}, T_{0}\right) \\
& \oplus \operatorname{Hom}_{\mathcal{H}}\left(T_{0}, T_{+}\right) \oplus \operatorname{Hom}_{\mathcal{H}}\left(T_{+}, T_{+}\right)
\end{aligned}
$$

is a quasi-tilted algebra. Thus in order to prove that $C$ is quasi-tilted it suffices to show that $K$ is the ideal generated by

$$
\operatorname{Hom}_{\mathcal{D}}\left(T_{-}, F T_{-}\right) \oplus \operatorname{Hom}_{\mathcal{D}}\left(T_{0}, F T_{-} \oplus F T_{0}\right) \oplus \operatorname{Hom}_{\mathcal{D}}\left(F^{-1} T_{+}, T_{0} \oplus T_{+}\right) .
$$

But this follows from the definition of $L$ and $R$ and the fact that the annihilators of the local slices $\Sigma_{L}$ and $\Sigma_{R}$ are given by the morphisms in $\operatorname{End}_{\mathcal{C}_{A}} T$ that factor through the lifts of the corresponding local slice in the cluster category. More precisely,

$$
\begin{aligned}
& \operatorname{Ann} \Sigma_{L} \cong \operatorname{Hom}_{\mathcal{D}}\left(F^{-1} T_{0} \oplus F^{-1} T_{+} \oplus T_{-}, T_{0} \oplus T_{+} \oplus F T_{-}\right), \\
& \operatorname{Ann} \Sigma_{R} \cong \operatorname{Hom}_{\mathcal{D}}\left(F^{-1} T_{+} \oplus T_{-} \oplus T_{0}, T_{+} \oplus F T_{-} \oplus F T_{0}\right),
\end{aligned}
$$

and thus

$$
\begin{aligned}
& \operatorname{Ann} \Sigma_{L} \cap \operatorname{Ann} \Sigma_{R} \cong \operatorname{Hom}_{\mathcal{D}}\left(T_{0}, F T_{0}\right) \oplus \operatorname{Hom}_{\mathcal{D}}\left(T_{-}, F T_{-}\right) \\
& \oplus \operatorname{Hom}_{\mathcal{D}}\left(F^{-1} T_{+}, T_{+}\right) \text {, }
\end{aligned}
$$


where we used the fact that $\operatorname{Hom}_{\mathcal{D}}\left(T_{-}, T_{+}\right)=\operatorname{Hom}_{\mathcal{D}}\left(T_{+}, T_{-}\right)=0$. This completes the proof that $C$ is quasi-tilted.

Since $C=\operatorname{End}_{\mathcal{H}} T$, we have $\widetilde{C}=\operatorname{End}_{\mathcal{C}_{\mathcal{H}}} T \cong \operatorname{End}_{\mathcal{C}_{A}} T=B$.

Now assume that $R=0$. Then $T_{-}=0$ and thus $K$ is generated by $\left(\right.$ Ann $\left.\Sigma_{L} \cap \operatorname{Ann} \Sigma_{R}\right) \oplus L$, and this is equal to

$$
\operatorname{Hom}_{\mathcal{D}}\left(T_{0}, F T_{0}\right) \oplus \operatorname{Hom}_{\mathcal{D}}\left(F^{-1} T_{+}, T_{+}\right) \oplus \operatorname{Hom}_{\mathcal{D}}\left(F^{-1} T_{+}, F T_{0}\right) .
$$

On the other hand, $T_{-}=0$ implies that

$$
\operatorname{Ann} \Sigma_{L}=\operatorname{Hom}_{\mathcal{D}}\left(F^{-1} T_{0} \oplus F^{-1} T_{+}, T_{0} \oplus T_{+}\right),
$$

and since $\operatorname{Hom}_{\mathcal{D}}\left(F^{-1} T_{0}, T_{+}\right)=0$, this implies that $K=\operatorname{Ann} \Sigma_{L}$ is the annihilator of a local slice. Therefore $C=B / K$ is tilted by [ABS2, Theorem 19]. The case where $L=0$ is proved in a similar way.

Conversely, assume $C$ is tilted. Then $K=\operatorname{Ann} \Sigma^{\prime}$ for some local slice $\Sigma^{\prime}$ in $\bmod B$. We show that $K=\operatorname{Ann} \Sigma_{L}$ or $K=\operatorname{Ann} \Sigma_{R}$. Suppose to the contrary that $\Sigma^{\prime}$ has both a predecessor and a successor in add $T_{0}$. Then there exists an arrow $\alpha$ in the quiver of $B$ such that $\alpha \in \operatorname{Hom}_{\mathcal{D}}\left(T_{0}, T_{0}\right)$ and $\alpha \in \operatorname{Ann} \Sigma^{\prime}=K$. But by definition of $\Sigma_{L}, \Sigma_{R}, L$ and $R$, we see that this is impossible.

Thus $K=\operatorname{Ann} \Sigma_{L}$ or $K=\operatorname{Ann} \Sigma_{R}$. In the former case, we have $R=0$, by the computation (6.1), and in the latter case, we have $L=0$.

Theorem 6.2. If $C$ is quasi-tilted of euclidean type and $B=\widetilde{C}$ then

$$
C=B / \operatorname{Ann}\left(\Sigma^{-} \oplus \Sigma^{+}\right),
$$

where $\Sigma^{-}$is a right section in the postprojective component of $C$ and $\Sigma^{+}$is a left section in the preinjective component.

Proof. $C$ being quasi-tilted implies that there is a hereditary category $\mathcal{H}$ with a tilting object $T$ such that $C=\operatorname{End}_{\mathcal{H}} T$. Moreover, $B=\operatorname{End}_{\mathcal{C}_{\mathcal{H}}} T$ is the corresponding cluster-tilted algebra. As before we use the decomposition $T=T_{-} \oplus T_{0} \oplus T_{+}$. Then the algebras

$$
C^{-}=\operatorname{End}_{\mathcal{H}}\left(T_{-} \oplus T_{0}\right) \quad \text { and } \quad C^{+}=\operatorname{End}_{\mathcal{H}}\left(T_{0} \oplus T_{+}\right)
$$

are tilted. Let $\Sigma^{-}$and $\Sigma^{+}$be complete slices in $\bmod C^{-}$and $\bmod C^{+}$respectively. Note that $\Sigma^{-}$lies in the postprojective component and $\Sigma^{+}$lies in the preinjective component of their respective module categories.

Then $C$ is a branch extension of $C^{-}$by the module

$$
M^{+}=\operatorname{Hom}_{\mathcal{H}}\left(T_{+}, T_{+}\right) \oplus \operatorname{Hom}_{\mathcal{H}}\left(T_{0}, T_{+}\right) .
$$

Similarly $C$ is a branch coextension of $C^{+}$by the module

$$
M^{-}=\operatorname{Hom}_{\mathcal{H}}\left(T_{-}, T_{-}\right) \oplus \operatorname{Hom}_{\mathcal{H}}\left(T_{-}, T_{0}\right) .
$$

For the definition of branch extension and branch coextension, see [Ri, Chapter 4]. Observe that the postprojective component of $C^{-}$does not change under the branch extension, and the preinjective component of $C^{+}$does not change under the branch coextension. Therefore $\Sigma^{-}$is a right section in the 
postprojective component of $C$ and $\Sigma^{+}$is a left section in the preinjective component. Moreover, by construction, we have

$$
\operatorname{Ann}_{B} \Sigma^{-}=M^{+} \oplus \operatorname{Ext}_{C}^{2}(D C, C) \text { and } \operatorname{Ann}_{B} \Sigma^{+}=M^{-} \oplus \operatorname{Ext}_{C}^{2}(D C, C),
$$

and therefore

$$
\operatorname{Ann}_{B}\left(\Sigma^{-} \oplus \Sigma^{+}\right)=\operatorname{Ann}_{B} \Sigma^{-} \cap \operatorname{Ann}_{B} \Sigma^{+}=\operatorname{Ext}_{C}^{2}(D C, C) .
$$

This completes the proof.

The main theorem of this section is the following.

Theorem 6.3. Let $C$ be a quasi-tilted algebra whose relation-extension $B$ is cluster-tilted of euclidean type. Then $C$ is one of the following.

(a) $C=B / \operatorname{Ann} \Sigma$ for some local slice $\Sigma$ in $\Gamma(\bmod B)$.

(b) $C=B / K$ for some partition ideal $K$.

Proof. Assume first that $C$ is tilted. Then, because of [ABS2, Theorem 19], there exists a local slice $\Sigma$ in the transjective component of $\Gamma(\bmod B)$ such that $B / \operatorname{Ann} \Sigma=C$. Otherwise, assume that $C$ is quasi-tilted but not tilted. Then, because of [LS], there exists a hereditary category $\mathcal{H}$ of the form

$$
\mathcal{H}=\mathcal{T}^{-} \vee \mathcal{C} \vee \mathcal{T}^{+}
$$

and a tilting object $T$ in $\mathcal{H}$ such that $C=\operatorname{End}_{\mathcal{H}} T$. Because of Theorem 6.1 we get $C=B / K$ where $K$ is the partition ideal induced by the given partition of $\mathcal{H}$.

Example 6.4. Let $B$ be the cluster-tilted algebra of type $\widetilde{\mathbb{E}}_{7}$ given by the quiver

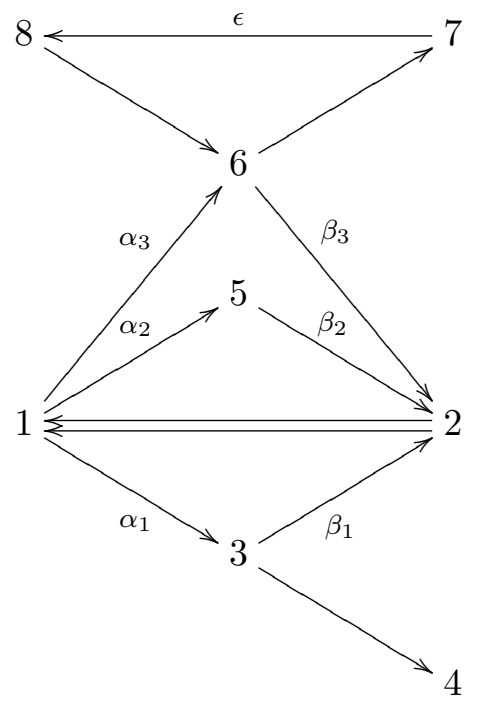

As usual let $T_{i}$ denote the indecomposable summand of $T$ corresponding to the vertex $i$ of the quiver. In this example $T$ has two transjective summands $T_{1}, T_{2}$, and the other summands lie in three different tubes. $T_{3}, T_{4}$ lie in a tube $\mathcal{T}_{1}, T_{5}$ lies in a tube $\mathcal{T}_{2}$ and $T_{6}, T_{7}, T_{8}$ lie in a tube $\mathcal{T}_{3}$. 
Choosing a partition ideal corresponds to choosing a subset of tubes to be predecessors of the transjective component. Thus there are 8 different partition ideals corresponding to the 8 subsets of $\left\{\mathcal{T}_{1}, \mathcal{T}_{2}, \mathcal{T}_{3}\right\}$. If the tube $\mathcal{T}_{i}$ is chosen to be a predecessor of the transjective component, then the arrow $\beta_{i}$ is in the partition ideal. And if $\mathcal{T}_{i}$ is not chosen to be a predecessor of the transjective component, then it is a successor and consequently the arrow $\alpha_{i}$ is in the partition ideal. The arrow $\epsilon$ is always in the partition ideal since it corresponds to a morphism from $T_{8}$ to $\mathrm{FT}_{7}$ in the derived category.

Summarizing, the 8 partition ideals $K$ are the ideals generated by the following sets of arrows.

$$
\left\{\alpha_{i}, \beta_{j}, \epsilon \mid i \notin I, j \in I, I \subset\{1,2,3\}\right\} .
$$

The quiver of the corresponding quasi-tilted algebra $B / K$ is obtained by removing the generating arrows from the quiver of $B$. Exactly 2 of these 8 algebras are tilted, and these correspond to cutting $\alpha_{1}, \alpha_{2}, \alpha_{3}, \epsilon$, respectively $\beta_{1}, \beta_{2}, \beta_{3}, \epsilon$.

\section{REFERENCES}

[Am] C. Amiot, Cluster categories for algebras of global dimension 2 and quivers with potential, Ann. Inst. Fourier 59 no 6, (2009), 2525-2590.

[A] I. Assem, Left sections and the left part of an Artin algebra, Colloq. Math. 116 (2009), no. 2, 273-300.

[ABCP] I. Assem, T. Brüstle, G. Charbonneau-Jodoin and P. G. Plamondon, Gentle algebras arising from surface triangulations. Algebra Number Theory 4 (2010), no. 2, 201-229.

[ABS] I. Assem, T. Brüstle and R. Schiffler, Cluster-tilted algebras as trivial extensions, Bull. Lond. Math. Soc. 40 (2008), 151-162.

[ABS2] I. Assem, T. Brüstle and R. Schiffler, Cluster-tilted algebras and slices, J. of Algebra 319 (2008), 3464-3479.

[ABS3] I. Assem, T. Brüstle and R. Schiffler, On the Galois covering of a cluster-tilted algebra, J. Pure Appl. Alg. 213 (7) (2009) 1450-1463.

[ABS4] I. Assem, T. Brüstle and R. Schiffler, Cluster-tilted algebras without clusters, J. Algebra 324, (2010), 2475-2502.

[AsScSe] I. Assem, R. Schiffler and K. Serhiyenko, Modules over cluster-tilted algebras that do not lie on local slices, preprint, arXiv:1606.05161.

[ASS] I. Assem, D. Simson and A. Skowronski, Elements of the Representation Theory of Associative Algebras, 1: Techniques of Representation Theory, London Mathematical Society Student Texts 65, Cambridge University Press, 2006.

[ARS] M. Auslander, I. Reiten and S.O. Smalø, Representation Theory of Artin Algebras Cambridge Studies in Advanced Math. 36, (Cambridge University Press, Cambridge, 1995).

[BT] M. Barot and S. Trepode, Cluster tilted algebras with a cyclically oriented quiver. Comm. Algebra 41 (2013), no. 10, 3613-3628.

[BFPPT] M. Barot, E. Fernandez, I. Pratti, M. I. Platzeck and S. Trepode, From iterated tilted to cluster-tilted algebras, Adv. Math. 223 (2010), no. 4, 1468-1494.

[BOW] M. A. Bertani-Økland, S. Oppermann and A Wrålsen, Constructing tilted algebras from cluster-tilted algebras, J. Algebra 323 (2010), no. 9, 2408-2428.

[BMRRT] A. B. Buan, R. Marsh, M. Reineke, I. Reiten and G. Todorov, Tilting theory and cluster combinatorics, Adv. Math. 204 (2006), no. 2, 572-618. 
[BMR] A. B. Buan, R. Marsh and I. Reiten, Cluster-tilted algebras, Trans. Amer. Math. Soc. 359 (2007), no. 1, 323-332 (electronic).

[BMR2] A. B. Buan, R. Marsh and I. Reiten, Cluster-tilted algebras of finite representation type, J. Algebra 306 (2006), no. 2, 412-431.

[CCS] P. Caldero, F. Chapoton and R. Schiffler, Quivers with relations arising from clusters $\left(A_{n}\right.$ case), Trans. Amer. Math. Soc. 358 (2006), no. 3, 1347-1364.

[FPT $]$ E. Fernández, N. I. Pratti and S. Trepode, On $m$-cluster tilted algebras and trivial extensions, J. Algebra 393 (2013), 132-141.

[FZ] S. Fomin and A. Zelevinsky, Cluster algebras I: Foundations, J. Amer. Math. Soc. 15 (2002), 497-529.

[H] D. Happel, A characterization of hereditary categories with tilting object. Invent. Math. 144 (2001), no. 2, 381-398.

[HRS] D. Happel, I. Reiten and S. Smal $\varnothing$, Tilting in abelian categories and quasitilted algebras. Mem. Amer. Math. Soc. 120 (1996), no. 575.

[HR] D. Happel and C. M. Ringel, The derived category of a tubular algebra, Proc. ICRA $I V$, Lecture Notes in Math., vol. 1177, Springer-Verlag, (1986) 156-180.

[LS] H. Lenzing and A. Skowroński, Quasi-tilted algebras of canonical type, Colloq. Math. 71 (1996), no. 2, 161-181.

[KR] B. Keller and I. Reiten, Cluster-tilted algebras are Gorenstein and stably CalabiYau, Adv. Math. 211 (2007), no. 1, 123-151.

[OS] M. Oryu and R. Schiffler, On one-point extensions of cluster-tilted algebras, em J. Algebra 357 (2012), 168-182.

[Re] I. Reiten, Cluster categories. Proceedings of the International Congress of Mathematicians. Volume I, 558-594, Hindustan Book Agency, New Delhi, 2010. 16-02

[Ri] C.M. Ringel, Tame Algebras and Integral Quadratic Forms, Lecture Notes in Math., vol. 1099, Springer-Verlag, 1984.

[R] C. M. Ringel, Representation theory of finite-dimensional algebras. Representations of algebras (Durham, 1985), 7-79, London Math. Soc. Lecture Note Ser., 116, Cambridge Univ. Press, Cambridge, 1986.

[S] R. Schiffler, Quiver Representations, CMS Books in Mathematics, Springer International Publishing, 2014.

[ScSe] R. Schiffler and K. Serhiyenko, Induced and coinduced modules over cluster-tilted algebras, to appear in J. Algebra, arXiv:1410.1732.

[ScSe2] R. Schiffler and K. Serhiyenko, Injective presentations of induced modules over cluster-tilted algebras, preprint, arXiv:1410.1732.

[Sk] A. Skowroński, Tame quasi-tilted algebras, J. Algebra 203 (1998), no. 2, 470-490.

Département de Mathématiques, Université de Sherbrooke, Sherbrooke, QuÉBEC, CANADA J1K 2R1

E-mail address: ibrahim.assem@usherbrooke.ca

Department of Mathematics, University of Connecticut, Storrs, CT 062693009, USA

E-mail address: schiffler@math.uconn.edu

Department of Mathematics, University of California, Berkeley, CA 947203840, USA

E-mail address: khrystyna.serhiyenko@berkeley.edu 